\title{
Interpretation of electron diffusion coefficient in organic and inorganic semiconductors with broad distributions of states
}

\author{
Juan Bisquert \\ Received 2nd January 2008, Accepted 26th February 2008 \\ First published as an Advance Article on the web 25th March 2008 \\ DOI: $10.1039 / b 719943 k$
}

The carrier transport properties in nanocrystalline semiconductors and organic materials play a key role for modern organic/inorganic devices such as dye-sensitized (DSC) and organic solar cells, organic and hybrid light-emitting diodes (OLEDs), organic field-effect transistors, and electrochemical sensors and displays. Carrier transport in these materials usually occurs by transitions in a broad distribution of localized states. As a result the transport is dominated by thermal activation to a band of extended states (multiple trapping), or if these do not exist, by hopping via localized states. We provide a general view of the physical interpretation of the variations of carrier transport coefficients (diffusion coefficient and mobility) with respect to the carrier concentration, or Fermi level, examining in detail models for carrier transport in nanocrystalline semiconductors and organic materials with the following distributions: single and two-level systems, exponential and Gaussian density of states. We treat both the multiple trapping models and the hopping model in the transport energy approximation. The analysis is simplified by thermodynamic properties: the chemical capacitance, $C_{\mu}$, and the thermodynamic factor, $\chi_{n}$, that allow us to derive many properties of the chemical diffusion coefficient, $D_{n}$, used in Fick's law. The formulation of the generalized Einstein relation for the mobility to diffusion ratio shows that the carrier mobility is proportional to the jump diffusion coefficient, $D_{J}$, that is derived from single particle random walk. Characteristic experimental data for nanocrystalline $\mathrm{TiO}_{2}$ in DSC and electrochemically doped conducting polymers are discussed in the light of these models.

\section{Introduction}

The subject of this paper is the interpretation of measured diffusion coefficient and mobilities for electronic transport in systems with a broad distribution of localized electronic states. Such systems include nanostructured metal-oxide semiconductors, and organic conductors, as well as more classical materials such as the amorphous inorganic conductors. These materials have raised increasing attention in the last two decades, in relation with applications such as dye-sensitized

Departament de Física, Universitat Jaume I, Castelló, 12071, Spain. E-mail: bisquert@fca.uji.es

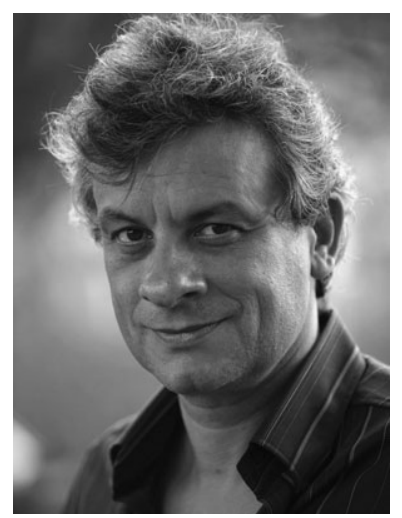

Juan Bisquert (Ph.D. 1992) is a professor of applied physics at Universitat Jaume I de Castello', where he leads the Group of Photovoltaic and Optoelectronic Devices. His recent research activity is focused on physical properties of nanoscale devices for clean energies, based on nanostructured metal-oxides and organic conductors, in particular dye-sensitized solar cells and organic LEDs. solar cells (DSC), ${ }^{1,2}$ organic solar cells,,${ }^{3,4}$ organic LEDs, ${ }^{5}$ organic electronics $^{6}$ and biological wiring. ${ }^{7}$

In the presence of a broad distribution of localized states, i.e., if the density of states (DOS) varies with energy over $0.5-1 \mathrm{eV}$ or more, electronic carriers are almost permanently localized. The transport occurs by carrier jumps either via a band of extended states, or if such states do not occur in a given material, directly via localized states. ${ }^{8}$ The transport coefficients are normally not constant quantities, but depend strongly on the Fermi level (or carrier concentration). Two main approaches have been used to describe such situations. The first is a classical multiple trapping transport. ${ }^{9-11}$ This model includes two classes of electronic states: the transport states above the mobility edge (that may be associated with extended states in the conduction band), and localized states in the bandgap. The latter states do not participate in spatial displacement but retain the carriers for a certain time by a trapping-detrapping process. The second approach is the hopping transport. ${ }^{12-18}$ Here, the transport occurs by transition between the states in the distribution, with a probability given by the Miller-Abrahams jump rate. ${ }^{19}$ Averaging the hopping rates over spatial and energy configurations is usually very difficult, but the analysis is partially simplified in a system with a steep distribution of localized states. For carriers situated deep enough energetically, a particular level, called the transport energy, $E_{\mathrm{tr}}$, determines the dominant hopping events. The occurrence of the effective transport level effectively reduces the hopping transport to multiple trapping, with $E_{\mathrm{tr}}$ playing the 
role of the mobility edge. The concept of transport energy was originally formulated ${ }^{20,21}$ for amorphous inorganic semiconductors with an exponential $\operatorname{DOS}^{17,20-23}$ and has been extended to organic conductors with a Gaussian DOS. ${ }^{15,17,24-26}$

When carrier transport is governed by activation to some type of transport level within a broad distribution of localized states, a great variation of the diffusion coefficient occurs as Fermi level moves in the bandgap, since the cost of promoting a carrier to the transport level is largely modified according to the occupation of the localized levels. The present work is motivated by the advent of new classes of disordered electronic materials of considerable technological relevance where the large variation of diffusion coefficient and mobility have been reported. In the organic conductors used in organic LEDs and FETs, Tanase et al. showed large dependence of the mobility on carrier density. ${ }^{27}$ In the DSC made with nanostructured $\mathrm{TiO}_{2}$ and liquid electrolyte, ${ }^{1}$ variations of diffusion coefficient were discovered by Peter and coworkers. ${ }^{28}$ Subsequently, it was shown that such variations could be related ${ }^{29}$ to the chemical capacitance of the nanostructured semiconductor. It was found that for the interpretation of the diffusion coefficient it is very important to distinguish between the chemical and jump diffusion coefficient. These notions, the chemical capacitance and the different diffusion coefficients, will feature prominently in the present paper. A striking demonstration of the difference between jump and kinetic diffusion coefficient was given by van de Lagemaat et al., ${ }^{30}$ and their work clearly showed the need for a careful identification of the diffusion coefficient that is being measured.

In the present work we are interested in quasi-equilibrium transport properties that determine steady-state device operation, and often provide sufficient knowledge of the transient behaviour as well. The central aim of this work is to facilitate the interpretation of experimental results about materials that are designed for their effectiveness as part of electronic and optoelectronic devices. ${ }^{31,32}$ These materials often display a large variability of properties, depending on conditions of preparation and measurement. Therefore, qualitative interpretation of the results for obtaining information on specific devices is often a priority of research. For this reason, in this paper we have attempted to describe fully the properties of the diffusion coefficient for activated transport in disordered materials, starting the application of the general formulae with very simple systems that already show some of the properties of the more complex ones. We usually represent the different quantities as a function of Fermi level. This is most appropriate for electrochemical and photoelectrochemical systems, where the Fermi level can be directly monitored by potentiostatic control. ${ }^{32-34}$ A system that played a key role in our understanding is the electrochemistry of redox polymers. ${ }^{35,36}$ In the work of Chidsey and Murray $^{35}$ the main concepts used here were clearly formulated. They identified the need to parametrize the varying chemical diffusion coefficient, and therefore introduced the chemical capacitance (called redox capacitance by them) and the conductivity-diffusivity relationship, eqn (20) below. So the spirit of the present work is an extension of ref. 35 including the energy disorder effects.

Another important aspect of electrochemical systems is that electron conduction in a solid phase surrounded by electrolyte (i.e., in nanostructured semiconductors, or conducting polymers, or redox hydrogels) is charge-compensated by ionic species. ${ }^{32}$ Eventually, the incorporation of ions may modify the intrinsic energy levels of the materials, as it will be commented in the case of electrochemical doping of polymers. ${ }^{37}$ In the present work we restrict our attention to the single-particle electronic DOS, consisting of a static distribution in the energy axis, and the interactions are neglected. ref. 32, 38-41 discuss additional examples of systems with strong interactions between carriers. The generalized Einstein relation for electron transport in arrays of quantum dots ${ }^{42}$ has already been adequately treated by van de Lagemaat, ${ }^{43}$ and many examples of transport in band semiconductors under degenerate conditions are analyzed in ref. 44; we do not treat these systems here in detail.

In section 2 we define the main thermodynamic properties and transport coefficients, the generalized Einstein relation, ${ }^{32}$ and the connection with experimental methods. In section 3 we give the general properties of the multiple trapping models. Section 4 presents two simple examples with discrete energy levels which illustrate the relevant physics. Thereafter we treat the carrier transport in the continuous DOS in examples that are important for the photovoltaic and optoelectronic devices: the exponential DOS (section 5), and the Gaussian disorder model (section 6).

Throughout these studies the analysis focuses on finding the effect of energy disorder on the main transport coefficients. We work with the assumption of spatial homogeneity that gives good results in many situations. This is a mean-field approach in which the distribution of electronic states in the energy axis is the same at every point, so that one can find typical transition/hopping probabilities by suitable averaging over energy and/or distance to the neighbor levels. However, it is clear that energy disorder is usually accompanied by spatial disorder. The prevalent paths for transport may take on special geometrical features, or may be circumscribed to restricted regions, if the distribution remains below the critical concentration for percolation. An extended treatment of these questions, that are briefly commented in section 7, is beyond the scope of the present report. We finish with some conclusions.

\section{Transport coefficients and chemical capacitance}

The connection between kinetic and transport properties outlined in this section is based on the phenomenological formulation of Reed and Ehrlich. ${ }^{45}$ This approach has been amply used in connection with surface diffusion, ${ }^{45}$ ion transport in solids ${ }^{46,47}$ and in the simulation of model systems consisting in interacting particles diffusing on the lattice. ${ }^{38,40,48}$ This formalism has recently been adapted ${ }^{32}$ for the interpretation of the electronic transport coefficients in electrochemical measurements in quasi-equilibrium conditions.

It should be remarked that the electrochemistry of ionically conducting solids has provided a wide experimental background that shows the necessity of these concepts and, in particular, the need to distinguish between the chemical and jump diffusion coefficient. A classical example is a huge increase of the thermodynamic factor in $\mathrm{Li}_{1+\delta} \mathrm{Al}$ alloy over a narrow potential range. ${ }^{49}$ Another example is that many of 
the curves reported below in Fig. 2 (chemical capacitance, thermodynamic factor, jump and chemical diffusion coefficients) are basically similar to those in ref. 50 for describing $\mathrm{Li}$ ion insertion into the graphite electrode (staged phase $3+4$ ) in terms of the two-level intercalation model.

\subsection{Chemical capacitance}

Let $E_{\mathrm{F}}$ be the electrochemical potential or Fermi level of the electrons, and $\mu$ their chemical potential. We have

$$
E_{\mathrm{F}}=-q \phi+\mu
$$

where $q$ is the elementary positive charge and $\phi$ is the local electrostatic potential. Here, we assume that the local electrostatic level (i.e., the conduction band position, $E_{\mathrm{c}}=-q \phi$ ) is not modified by variation of the Fermi level. Therefore a displacement of the Fermi level can be identified with a variation of the chemical potential, $\mathrm{d} E_{\mathrm{F}}=\mathrm{d} \mu$. The chemical capacitance (per unit volume) is defined as ${ }^{51,52}$

$$
C_{\mu}=q^{2} \frac{\mathrm{d} n}{\mathrm{~d} \mu}
$$

The same quantity was introduced in ref. 35 as a redox capacitance. Assuming a DOS function $g(E)$, the carrier density is found as

$$
n=\int_{-\infty}^{+\infty} g(E) f\left(E-E_{\mathrm{F}}\right) \mathrm{d} E
$$

where $f\left(E-E_{\mathrm{F}}\right)$ is the Fermi-Dirac function,

$$
f\left(E-E_{\mathrm{F}}\right)=\frac{1}{1+e^{\left(E-E_{\mathrm{F}}\right) / k_{\mathrm{B}} T}}
$$

that was reduces to the Boltzmann distribution, $f(E)=$ $e^{-\left(E-E_{\mathrm{F}}\right) / k_{\mathrm{B}} T}$, when $E-E_{\mathrm{F}} \gg k_{\mathrm{B}} T$, with $k_{\mathrm{B}}$ being Boltzmann's constant and $T$ the absolute temperature. The chemical capacitance is

$$
\begin{aligned}
C_{\mu} & =-q^{2} \int_{-\infty}^{+\infty} g(E) \frac{\mathrm{d} f}{\mathrm{~d} E_{\mathrm{F}}}\left(E-E_{\mathrm{F}}\right) \mathrm{d} E \\
& =\frac{q^{2}}{k_{\mathrm{B}} T} \int_{-\infty}^{+\infty} g(E) f\left(E-E_{\mathrm{F}}\right)\left[1-f\left(E-E_{\mathrm{F}}\right)\right] \mathrm{d} E
\end{aligned}
$$

In the zero-temperature limit, the capacitance is related to the DOS function as ${ }^{53}$

$$
C_{\mu}=q^{2} g\left(E_{\mathrm{F}}\right)
$$

In this approximation, the Fermi-Dirac function is a unity step function at the Fermi level. Therefore, displacing the Fermi level by $\mathrm{d} E_{\mathrm{F}}$ simply fills with carriers a slice of the DOS: $\mathrm{d} n=g\left(E_{\mathrm{F}}\right) \mathrm{d} E_{\mathrm{F}}$.

\subsection{Diffusion coefficients}

The random walks of an electronic carrier determine the jump diffusion coefficient, that has the form ${ }^{40,48}$

$$
D_{J}=\frac{1}{6 t}\left\langle\left(\frac{1}{N} \sum_{i=1}^{N} \Delta r_{i}\right)^{2}\right\rangle
$$

where $\Delta r_{i}$ is the displacement of the $i$ th particle at time $t$, and \langle\rangle denotes a statistical average. More precisely, the jump (or kinetic) diffusion coefficient defined by eqn (7a) reflects diffusion of the center of mass of $N$ particles, while the tracer diffusion coefficient, $D^{*}$, reflects random walks of a particle

$$
D^{*}=\lim _{t \rightarrow \infty} \frac{1}{6 N t}\left\langle\sum_{i=1}^{N}\left(\Delta r_{i}\right)^{2}\right\rangle
$$

If on average, there are no cross correlations between displacements $\Delta r_{i}(t)$ of different particles at different times, $D_{J}$ and $D^{*}$ become equivalent. ${ }^{40,48}$ Monte Carlo simulations show that jump and tracer diffusion coefficient are practically identical in many conditions. ${ }^{54}$ The jump diffusion coefficient can often be expressed as $38,39,45$

$$
D_{\mathrm{J}}=\langle\nu\rangle\left\langle r^{2}\right\rangle
$$

in terms of a mean effective jump frequency $\langle\nu\rangle$, and the square of effective jump length $\left\langle r^{2}\right\rangle$. Eqn (8) also takes a numerical prefactor of order 1 depending on the dimensionality.

On the other hand, experimental information on the fundamental jump rates is often derived from the chemical diffusion coefficient, $D_{n}$, that relates the flux $J_{n}$ to the gradient of the concentration by Fick's law

$$
J_{n}=-D_{n} \frac{\partial n}{\partial x}
$$

The diffusion coefficients $D_{n}$ and $D_{\mathrm{J}}$ differ by the quantity $\chi_{n},{ }^{39,45,48}$

$$
D_{n}=\chi_{n} D_{\mathrm{J}}
$$

that is called the thermodynamic factor, ${ }^{55}$ and is defined as follows

$$
\chi_{n}=\frac{n}{k_{\mathrm{B}} T} \frac{\partial \mu}{\partial n}
$$

For the Boltzmann statistics $\chi_{n}=1$, and there is no difference between jump and diffusion coefficient, but when the statistics of electrons differs from ideality, $\chi_{n}$ can differ largely from 1. In general, both site-saturation effects (as discussed below) and interactions ${ }^{32,38-41}$ cause a variation of $\chi_{n}$. $\chi_{n}$ can also be expressed with respect to the chemical capacitance as

$$
\chi_{n}=\frac{q^{2} n}{k_{\mathrm{B}} T} \frac{1}{C_{\mu}}
$$

\subsection{Generalized Einstein relation}

In the electron transport in non-degenerate band-conduction materials, a single transport level consisting of extended states is well defined. The conductivity is given by

$$
\sigma_{n}=q n u_{n}
$$

where $n$ is the total density of electrons and $u_{n}$ is the electron mobility. The mobility $u_{n}$ and the diffusion coefficient $D_{n}$ are constant quantities and satisfy the standard Einstein relation

$$
\frac{D_{n}}{u_{n}}=\frac{k_{\mathrm{B}} T}{q}
$$


It has long been recognized that eqn (14) has important limitations. For band transport in semiconductors, it holds only under Boltzmann distribution, i.e. when the chemical potential of electrons satisfies $\mu=k_{\mathrm{B}} T \ln n$. The generalized Einstein relationship has the form ${ }^{56,57}$

$$
\frac{D_{n}}{u_{n}}=\frac{1}{q} \frac{n}{(\mathrm{~d} n / \mathrm{d} \mu)}
$$

The subject of application of mobility-diffusion relation in disordered conductors became of interest in the early 1990s. Deviations from eqn (14) were found, first, by Monte Carlo simulation of hopping drift and diffusion of carriers in a lattice with an array of hopping sites with a Gaussian distribution of site energies. ${ }^{58-60}$ This was observed experimentally in hole transport in 1,1-bis(di-4-tolylaminophenyl)cyclohexane. ${ }^{61}$ Deviations were also found by $\mathrm{Gu}$ et al. in measurements of transport in hydrogenated amorphous silicon with exponential DOS. ${ }^{62,63}$ However, the interpretation of experimental results was not clearly established at that time due to the complex carrier distribution in the time-of-flight technique. ${ }^{64}$ The Einstein relation for transport in the exponential density of states (DOS) was first analyzed by Ritter et al. ${ }^{65}$ For the Gaussian DOS, it was applied by Roichman and Tessler ${ }^{66}$ and by other authors. ${ }^{6-73}$ The current understanding of this subject has been recently summarized. ${ }^{74}$

However, eqn (15) is very often referred to in solid-state physics textbooks, ${ }^{75,76}$ where the generalized Einstein relation is developed for degenerate semiconductors. Thus the peculiarities of the broad densities of states, and the possibility of separately measuring the (chemical) diffusion coefficient and mobility, were not addressed, and there has been confusion in the past, regarding which the diffusion coefficient entering in eqn (15) is. This is discussed in the Appendix. It is the opinion of the present author that such confusion is removed by distinguishing the chemical and jump diffusion coefficient. Therefore we use the following formulation of the generalized Einstein relation, that is discussed in a recent paper. ${ }^{32}$ In terms of the chemical diffusion coefficient we have

$$
\frac{D_{n}}{u_{n}}=\chi_{n} \frac{k_{\mathrm{B}} T}{q}
$$

This is another statement of eqn (15), see the Appendix. The definition of the mobility is given in terms of the average carrier velocity $\langle v(F)\rangle$ acquired under electrical field $F$, at low field values.

$$
u_{n}=\left.\frac{\mathrm{d}\langle v(F)\rangle}{\mathrm{d} F}\right|_{F=0}
$$

The mobility can also be defined on the basis of the difference of effective charge carrier jump probability in the direction along and against the electric field. ${ }^{77}$ From eqns (10) and (16), $u_{n}$ is proportional to the jump diffusion coefficient

$$
u_{n}=\frac{q D_{J}}{k_{\mathrm{B}} T}
$$

Eqn (18) is generally valid and has the form of the classical Einstein relationship in eqn (14). However, as has already been stated, $D_{\mathrm{J}}$ is not in general the diffusion coefficient appearing in Fick's law.
Eqn (18) is, of course, not new: it is routinely used for example in analytic models and Monte Carlo simulations of hopping transport in disordered materials, which are normally restricted to the random walk of a single carrier. ${ }^{78}$ In calculations of hopping theory, it is a standard procedure to average over spatial and energy configurations in order to find the effective jump frequency and length that allow to calculate the jump diffusion coefficient using eqn (8), and immediately the mobility using eqn (18), ${ }^{78,79}$ see the Appendix for further discussion.

The conductivity in eqn (13) can be expressed as

$$
\sigma_{n}=q^{2} D_{n} \frac{\mathrm{d} n}{\mathrm{~d} \mu}
$$

The conductivity can also be written as ${ }^{34,80}$

$$
\sigma_{n}=D_{n} C_{\mu}
$$

Eqn (20) is suggested in ref. 35 as a definition of the diffusion coefficient. However, we have shown that $D_{n}$ in eqn (20) is the chemical diffusion coefficient that can be separately defined. Therefore the conductivity-diffusivity relationship (20) is most appropriately viewed as a direct expression of the generalized Einstein relationship. One should remark that all three quantities contained in eqn (20) (conductivity, chemical diffusion coefficient, and chemical capacitance) are distinctly measurable with electrochemical methods.

Some authors use an alternative expression for the mobility that relates to the chemical diffusion coefficient. ${ }^{42,57,81-83}$ It is assumed that in a quasi-equilibrium situation only the electrons within $k_{\mathrm{B}} T$ of the Fermi level contribute significantly to conductivity. The effective density of carriers $\hat{n}$ is given by ${ }^{57}$

$$
\hat{n}=k_{\mathrm{B}} T \frac{\mathrm{d} n}{\mathrm{~d} \mu}=\frac{n}{\chi_{n}}
$$

Then one can define an effective carrier mobility from the conductivity

$$
\sigma_{n}=q \hat{n} \hat{u}_{n}
$$

Using eqn (13), we obtain

$$
\hat{u}_{n}=\frac{q D_{n}}{k_{\mathrm{B}} T}
$$

It should be noticed that $u_{n}$ and $\hat{u}_{n}$ differ by the thermodynamic factor, $\chi_{n}, c f$. eqn (18).

\subsection{Experimentally measured quantities}

Before we enter the revision of particular transport models in certain DOS, let us discuss the meaning of the different quantities found from experiments. Our results relate only to systems that are close to equilibrium, i.e. the carriers in all the states of the distribution are thermalized to a steady-state Fermi level. We also adopt the quasistatic approximation, ${ }^{80}$ meaning that the time for trapping-detrapping is substantially shorter than the characteristic transit time across the sample. Measurements are made by a small perturbation (indicated by $\Delta$ ) of different quantities. This procedure is routinely used in electrochemical and photoelectrochemical systems, where the electrode potential is proportional to electrons (or holes) Fermi level. ${ }^{32}$ We do not list here all the different techniques, 
but only the essential points they have in common, see ref. 32 for a more general explanation.

(1) The conductivity is measured as a relation of electrical current $\Delta I$ to voltage $\Delta V$. This can be done at steady-state by electrochemical gating, ${ }^{84,85}$ or as the low-frequency resistance in impedance spectroscopy (IS). ${ }^{86}$

(2) The chemical capacitance is measured as a relation of charge $\Delta Q$ to voltage $\Delta V$, when the voltage displaces the Fermi level. This can be done by step charging or else obtaining the low-frequency capacitance from IS. ${ }^{33}$ In many cases eqn (6) is a good approximation to eqn (5) and then the DOS is directly measured by the chemical capacitance. However, this is not always true, for example eqn (6) gives incorrect results at low carrier densities in a Gaussian DOS, as discussed later. In general, the DOS cannot be measured directly, but has to be deconvoluted in eqn (6) from $C_{\mu}$, that is the measured quantity. ${ }^{87}$ Alternatively, the DOS can be obtained from thermally stimulated current (TSC) methods, which also require demerging the experimental signal. ${ }^{88}$ On the other hand, the carrier density $n$ can be found readily integrating the chemical capacitance with respect to voltage, eqn (2).

(3) The chemical diffusion coefficient of electrons, $D_{n}$, is directly measured by transient methods (either in time or frequency domain) such as IS. ${ }^{29}$ The determination of $D_{n}$ consists of inducing a disequilibrium by a voltage step, $\Delta V$, and taking the time constant for equilibration, that relates to the transit time for diffusion across the sample. These methods are very common in the electrochemistry of ionic conductors, see, e.g., ref. 89 and 90 .

(4) In electrochemical systems the mobility is found from the conductivity and the carrier density by eqn (13). ${ }^{91}$ In organic conductors it is often measured in the space-charge limited conduction regime, or by time-of-flight method. Eqn (20) indicates that if one divides the conductivity by the chemical capacitance, the resulting quantity is not the mobility, but the chemical diffusion coefficient.

\section{Multiple trapping models}

As mentioned in the Introduction, multiple trapping models are based on a net distinction between the role of electronic states above and below a mobility edge. This distinction is in accord with the classical semiconductor physics, where all localized states in the bandgap, below the conduction band edge, are traps. In organic conductors, the width of the bands are very narrow and extended states are rarely observed. ${ }^{88}$ Nonetheless in systems dominated by hopping between localized states, multiple trapping transport is recovered to a certain extent with the concept of the transport energy, as discussed below in more detail. In this section we treat the multiple trapping models proper, and we derive a set of general relationships that are valid for any particular distribution of traps and transport mechanism.

Multiple trapping model entails by definition the effect of some trap levels over the rate of displacement through transport states. Such an effect can be described by the full set of transport-kinetic equations of the model, that provides the system's response in any required set of conditions. However, if trapping and detrapping are fast processes, then electron trapping kinetics can be readily described in terms of electron densities in transport and trap states, and this second approach will be adopted herein. ${ }^{80}$ This is a consequence of the principle of detailed balance, ${ }^{92}$ that links the kinetic constants for trapping and detrapping to the equilibrium occupancies. ${ }^{80}$ A recent, general analysis ${ }^{93}$ shows that the first, general, approach reduces to the second one whenever the traps can be considered in quasi-equilibrium conditions.

\subsection{General relationships}

We call the transport states a set of states where spatial displacement of carriers occurs, with a DOS $g_{0}(E)$, total number $N_{0}$, number of carriers $n_{0}$, and the chemical capacitance $C_{\mu}^{0}$. The transport mechanism can consist of band transport, hopping, etc. Whatever the case, the displacement of electrons is characterized by an effective jump frequency $\left\langle\nu_{0}\right\rangle$, a jump diffusion coefficient, $D_{\mathrm{J}}^{0}$, and the resulting chemical diffusion coefficient $D_{n}^{0}$,

$$
D_{n}^{0}=\left(\frac{q^{2} n_{0}}{k_{\mathrm{B}} T} \frac{1}{C_{\mu}^{0}}\right) D_{\mathrm{J}}^{0}
$$

All these transport coefficients related to the transport states may depend on the Fermi level (or carrier density). The second element of a multiple trapping model is a distribution of deeper localized states (below the mobility edge), $g_{\mathrm{L}}(E)$, with the total number $N_{\mathrm{L}}$, the number of carriers $n_{\mathrm{L}}$, and the chemical capacitance $C_{\mu}^{\mathrm{L}}$. There is no hopping between the states in the deeper levels; these states only trap and release the carriers in the transport levels. Note the relationships

$$
\begin{aligned}
n & =n_{0}+n_{\mathrm{L}} \\
C_{\mu} & =C_{\mu}^{0}+C_{\mu}^{\mathrm{L}} \\
& =C_{\mu}^{0}\left(1+\frac{\partial n_{\mathrm{L}}}{\partial n_{0}}\right)
\end{aligned}
$$

for the total carrier density and total chemical capacitance, respectively.

The central kinetic relationship in the multiple trapping models is the following

$$
n\langle\nu\rangle=n_{0}\left\langle\nu_{0}\right\rangle
$$

where $\langle\nu\rangle$ is the average jump frequency for all the carriers. Eqn (27) uses the quasi-static approximation (so that the trap dynamics is removed) and expresses the average number of transitions in the transport levels either in terms of carriers in the transport levels or in terms of all the carriers in the system. It follows from eqn (27) that the jump diffusion coefficient relates to $D_{\mathrm{J}}^{0}$ as

$$
D_{\mathrm{J}}=\frac{n_{0}}{n} D_{\mathrm{J}}^{0}
$$

Using eqns (12) and (26), the thermodynamic factor can be written as

$$
\chi_{n}=\frac{q^{2} n}{k_{\mathrm{B}} T} \frac{1}{C_{\mu}^{0}}\left(1+\frac{\partial n_{\mathrm{L}}}{\partial n_{0}}\right)^{-1}
$$


Hence, the chemical diffusion coefficient has the general form $^{29}$

$$
\begin{aligned}
D_{n} & =\left(1+\frac{\partial n_{\mathrm{L}}}{\partial n_{0}}\right)^{-1}\left(\frac{q^{2} n_{0}}{k_{\mathrm{B}} T} \frac{1}{C_{\mu}^{0}}\right) D_{\mathrm{J}}^{0} \\
& =\left(1+\frac{\partial n_{\mathrm{L}}}{\partial n_{0}}\right)^{-1} D_{n}^{0}
\end{aligned}
$$

where we have applied eqn (24) in the last equality. Alternatively, we can write eqn (30) as

$$
D_{n}=\frac{C_{\mu}^{0}\left(E_{\mathrm{F}}\right)}{C_{\mu}^{0}\left(E_{\mathrm{F}}\right)+C_{\mu}^{L}\left(E_{\mathrm{F}}\right)} D_{n}^{0}
$$

The effect of trapping in the chemical diffusion coefficient is dominant when $\partial n_{\mathrm{L}} / \partial n_{0} \gg 1$. In this case the result is

$$
D_{n}=\left(\frac{\partial n_{0}}{\partial n_{\mathrm{L}}}\right) D_{n}^{0}
$$

which can also be expressed

$$
D_{n}=\frac{C_{\mu}^{0}\left(E_{\mathrm{F}}\right)}{C_{\mu}^{L}\left(E_{\mathrm{F}}\right)} D_{0}
$$

Using eqns (20) and (31) we obtain the following result for the conductivity:

$$
\sigma_{n}=D_{n} C_{\mu}=D_{n}^{0} C_{\mu}^{0}
$$

From eqns (13), (16) and (28) we can also express the conductivity in terms of the carrier density and jump diffusion coefficient

$$
\sigma_{n}=\frac{q^{2} n}{k_{\mathrm{B}} T} D_{\mathrm{J}}=\frac{q^{2} n_{0}}{k_{\mathrm{B}} T} D_{\mathrm{J}}^{0}
$$

Note that the quantities in the last terms of eqns (34) and (35) depend only on the properties of the transport states.

\subsection{Interpretation of multiple trapping models}

Let us discuss the general implications of these results for the interpretation of transport in multiple trapping systems:

(1) Eqn (28) indicates the classical result ${ }^{94}$ that the mobility in the presence of the traps is reduced by a factor corresponding to the proportion of carriers in the transport states to total number of carriers.

(2) Eqns (32) and (33) show that the chemical diffusion coefficient in the presence of traps is reduced by the relationship of free to trapped number of electrons for a small variation of the Fermi level. This prefactor describes the delay of response of the chemical diffusion coefficient (in the quasistatic approximation ${ }^{80}$ ), with respect to the free electrons diffusion coefficient (in transport states), by the trapping and detrapping process. ${ }^{80,95}$ Such delay is unavoidable when measuring the chemical diffusion coefficient by any transient technique, since the release of trapped carriers introduces additional time to reach quasi-stationary conditions.

(3) Eqn (34) shows that the conductivity is determined exclusively by the transport level and is completely independent of the presence and distribution of traps. The steady-state conduction is not affected by the trapping process, because the traps remain in equilibrium. Alternatively, one can view conduction as the result of the displacement of the whole electron density, $n$, with a smaller jump diffusion coefficient, eqn (35). However, it should also be remarked that the introduction of traps can have important effects in the steady-state conduction process. The charged traps modify substantially the electrical field distribution, which dominates the steady-state drift currents in OLEDs, for example. On the other hand, in practice the introduction of dopants in organic systems may modify the transport states, by reducing their energy, and in this case the conductivity will be modified. ${ }^{88}$ Finally, in a system with very slow traps, the conductivity depends heavily on the time constants of the traps, as discussed in detail elsewhere. ${ }^{93}$

\subsection{Band transport}

To conclude the general analysis of multiple trapping models, we consider the usual situation in which the transport level $E_{0}$ consists of the lower edge of the conduction band, while the trap states are situated below, in the bandgap. If we restrict our attention to the domain of potentials in which the Fermi level remains below $E_{0}$, so that we avoid degeneracy effects, the free carriers in extended states are well described by Boltzmann statistics. Eqn (35) can be simplified as

$$
\chi_{n}=\frac{n}{n_{0}} \frac{1}{1+\frac{\partial n_{\mathrm{L}}}{\partial n_{0}}}
$$

In eqn (36) we can separate two cases. If the free carriers are dominant (which usually occurs when the Fermi level approaches the conduction band) then $\chi_{n}=1$. According to eqn (40), below, the term in parentheses in eqn (24) is 1, and we have $D_{n}^{0}=D_{\mathrm{J}}^{0}=\bar{D}_{0}$ for the free carriers. On the other hand, if the traps are dominant, then

$$
\chi_{n}=\frac{n_{\mathrm{L}}}{n_{0}} \frac{\partial n_{0}}{\partial n_{\mathrm{L}}}
$$

This last equation can be written in terms of derivatives of chemical potentials for free and localized carriers:

$$
\chi_{n}=\frac{\partial \mu_{0}}{\partial \mu_{\mathrm{L}}}
$$

\section{Simple models with discrete levels}

\subsection{Transport by hopping in a single level}

The first application we discuss is the transport of electrons by hopping between neighbor localized sites of a unique energy level $E_{0}$ with a volume density $N_{0}$. Transport coefficients in this model are given in ref. 29 and the Einstein relation is discussed in ref. 96. We neglect percolation effects that are discussed in section 7. The chemical capacitance has the value

$$
C_{\mu}=\frac{N_{0} q^{2}}{k_{\mathrm{B}} T} f(1-f)
$$

in terms of the occupancy $f=n / N_{0}$. In the dilute limit $(f \ll 1)$, corresponding to Boltzmann distribution, eqn (39) gives

$$
C_{\mu}=\frac{q^{2} n}{k_{\mathrm{B}} T}=\frac{q^{2} N_{0}}{k_{\mathrm{B}} T} \exp \left[-\left(E_{0}-E_{\mathrm{F}}\right) / k_{\mathrm{B}} T\right]
$$



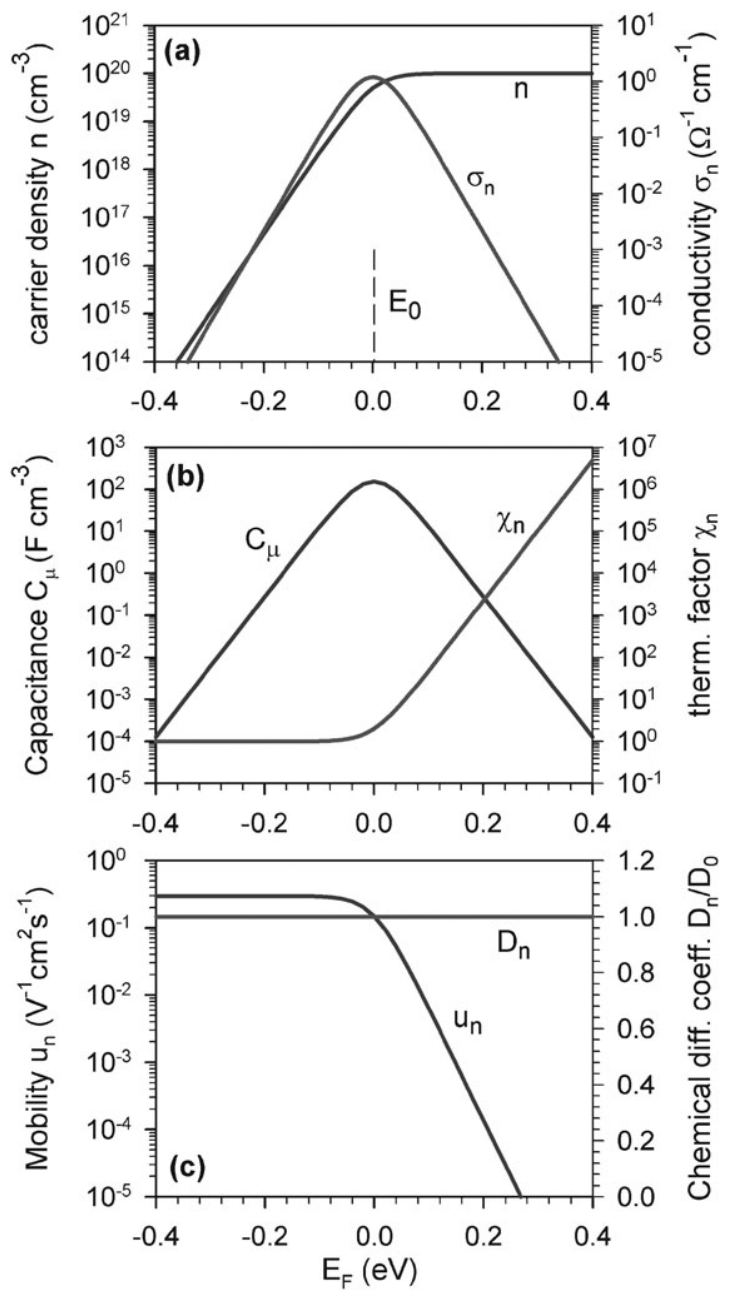

Fig. 1 Representation of several quantities for charge accumulation and transport by hopping between localized states of a single energy $E_{0}=0 \mathrm{eV} . E_{\mathrm{F}}$ is the Fermi level potential. (a) Carrier density and conductivity. (b) Chemical capacitance and thermodynamic factor. (c) Mobility and chemical diffusion coefficient. The following parameters were used in the calculation: $N_{0}=1.0 \times 10^{20} \mathrm{~cm}^{-3}, T=300 \mathrm{~K}$, $\nu_{0}=10^{12} \mathrm{~s}^{-1}, a=10^{-7} \mathrm{~cm}$.

The general shape of the capacitance, shown in Fig. 1a, forms a peak at the potential $E_{\mathrm{F}}=E_{0}$, at which the occupancy $f=1 / 2$. The mean effective jump frequency is

$$
\langle\nu\rangle=\bar{v}_{0}(1-f)
$$

where $\bar{v}_{0}$ is the rate constant for hopping from an occupied site to an empty site at the distance $R=\left(N_{0}\right)^{-1 / 3}$. The jump diffusion coefficient is

$$
D_{\mathrm{J}}=\frac{\langle\nu\rangle}{N_{0}^{2 / 3}}
$$

and the mobility has the form

$$
u_{n}\left(E_{\mathrm{F}}\right)=\frac{q \bar{\nu}_{0}}{k_{\mathrm{B}} T N_{0}^{2 / 3}}(1-f)
$$

The chemical diffusion coefficient is a constant, $D_{n}=\bar{D}_{0}=$ $\bar{v}_{0} R^{2}$. There is a strong difference between mobility and diffusivity due to the exclusion effect, see Fig. 1c. Since $u_{n}$ relates to the random walk displacement of electrons, it decreases when carrier density is high, due to the fact that hopping probability decreases when the neighbor sites becoming occupied, reduces, eqn (43). In contrast to this, the chemical diffusion coefficient describes the net flux under a gradient of the concentration, and in this case, the exclusion effects of forward and backward jumps between two neighbor sites counterbalance, giving the constant $D_{n}$, as explained in ref. 45. Central to our discussion is the fact that this difference between mobility and diffusion coefficient is completely described by the thermodynamic factor, as indicated in eqn (16). In the present example it is

$$
\chi_{n}=1 /(1-f)
$$

see Fig. 1b.

A relevant instance of the difference between mobility and chemical diffusion coefficient is found in the study ${ }^{43}$ of electron transport in an array of quantum dots with a series of discrete energy levels. By filling the $1 \mathrm{~S}_{0}$ level, the diffusion coefficient decreases by a factor of 10 , while the mobility shows a much stronger decay by three orders of magnitude, which is observed in measurements. ${ }^{97}$

Returning to our single-level example, the peak of the conductivity, shown in Fig. 1b, can be explained by the combined behaviors of carrier density and mobility. At low Fermi level the mobility is constant and the conductivity increases with the increase of carrier density. Above $E_{\mathrm{F}}=E_{0}$ the density of electrons is $\approx N_{0}$, but then, the mobility starts to decrease because most of the transport states have been occupied. As a result, the conductivity has the same shape as the chemical capacitance, which is summarily expressed in eqn (20).

\subsection{Two-level (single trap) model}

The simplest model of trap-limited transport is composed of a transport level at energy $E_{0}$ (for which we take the hopping model described in section 4.1), and a trap level at energy $E_{1}$ with volume density $N_{1}=\delta N_{0}$, where $\delta$ is a constant, see Fig. 2. The model is discussed in ref. 98. In equilibrium the occupancies of the two levels are determined by the equations

$$
E_{F}=E_{0}+k_{\mathrm{B}} T \ln \left(\frac{f_{0}}{1-f_{0}}\right)=E_{1}+k_{\mathrm{B}} T \ln \left(\frac{f_{1}}{1-f_{1}}\right)
$$

When the Fermi level increases, the two states are consecutively filled up with carriers, Fig. 2a. Consequently, there are two peaks in the chemical capacitance that corresponds to the addition of two terms as those in eqn (39), one for each level, ${ }^{98}$ see Fig. 2b. The chemical diffusion coefficient is obtained from eqn (31)

$$
D_{n}=\frac{\bar{D}_{0}}{1+\delta \frac{f_{1}\left(1-f_{1}\right)}{f_{0}}}
$$

When the Fermi level lies deep below the trap state $E_{1}$, both $E_{0}$ and $E_{1}$ are populated following the ideal statistics $\left(f_{0}, f_{1} \ll 1\right)$. Hence the thermodynamic factor is 1 , Fig. $2 \mathrm{~b}$, and the mobility and chemical diffusion coefficient in eqn (46) take 

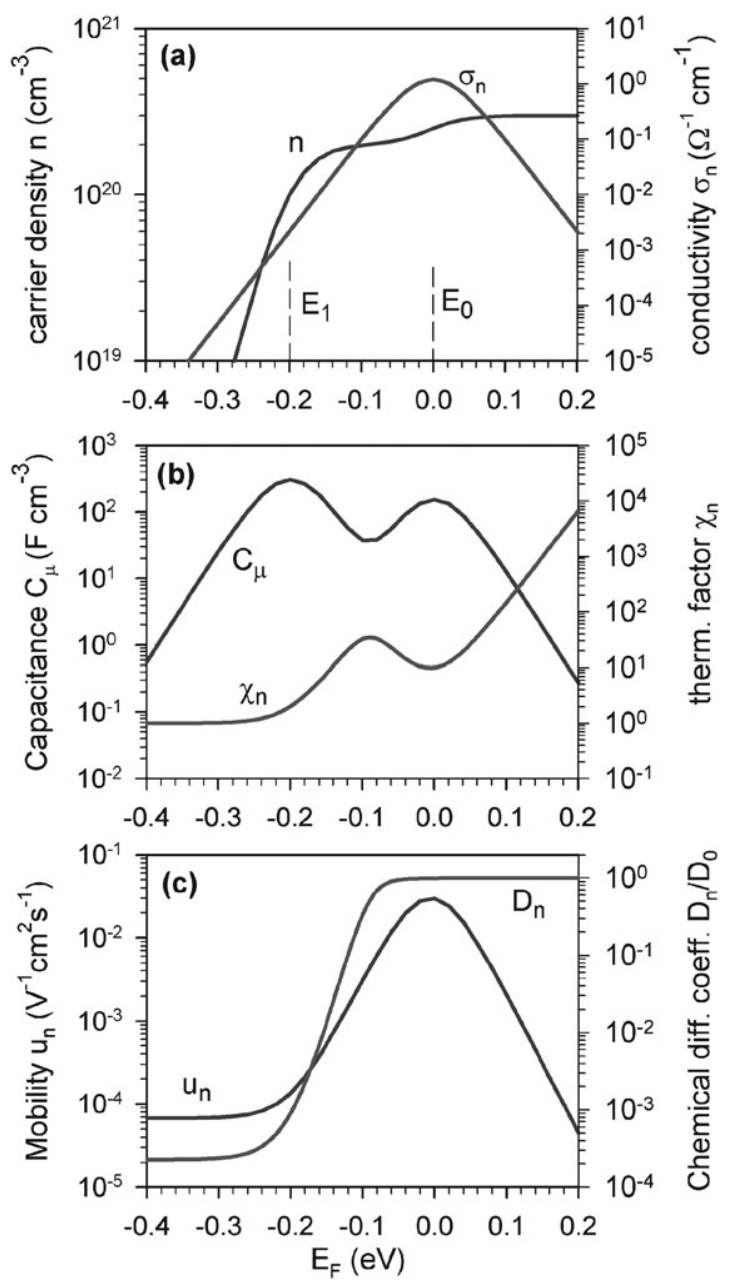

Fig. 2 Representation of several quantities for charge accumulation and transport by hopping between localized states in a material with a single bandgap state of energy $E_{0}=0 \mathrm{eV}$, and a trap level at $E_{1}=$ $-0.2 \mathrm{eV} . E_{\mathrm{F}}$ is the Fermi level potential. (a) Carrier density and conductivity. (b) Chemical capacitance and thermodynamic factor. (c) Mobility and chemical diffusion coefficient. The following parameters were used in the calculation: $N_{0}=1.0 \times 10^{20} \mathrm{~cm}^{-3}, N_{1}=2 N_{0}$, $T=300 \mathrm{~K}, \nu_{0}=10^{12} \mathrm{~s}^{-1}, a=10^{-7} \mathrm{~cm}$.

constant values

$$
D_{n}=\frac{\bar{D}_{0}}{1+\delta \exp \left[\left(E_{0}-E_{1}\right) / k_{\mathrm{B}} T\right]}
$$

This result is known as the Hoesterey-Letson formula, from their model developed for doped anthracene crystals. ${ }^{11}$ Changes in $D_{n}$ and $u_{n}$ appear when the deep states begin to be more heavily occupied. Filling the deep traps reduces their slowing down effect, hence the chemical diffusion coefficient increases rapidly, Fig. 2c, until the deep state is filled completely, at which point the chemical diffusion coefficient becomes a constant identical to the single level case (section 4.1). The mobility (related to the jump diffusion coefficient), shows an additional feature: the decrease at high carrier density due to the occupation of the transport states, already described in section 4.1. While the trap state affects severely both $D_{n}$ and $u_{n}$ with respect to the trap-free case, it should be noticed that the conductivity, shown in Fig. 2a, is not changed at all with respect to Fig. 1a. This is expected as explained in section 3.2.

\section{Carrier transport in exponential DOS}

\subsection{Multiple trapping in exponential DOS}

An exponential distribution of localized states in the bandgap, usually applied in amorphous semiconductors, has the expression

$$
g_{\mathrm{L}}(E)=\frac{N_{\mathrm{L}}}{k_{\mathrm{B}} T_{0}} \exp \left[\left(E-E_{0}\right) / k_{\mathrm{B}} T_{0}\right]
$$

where $N_{\mathrm{L}}$ is the total density and $T_{0}$, is a parameter with temperature units that determines the depth of the distribution below the transport level $E_{0}$. The main features of this model are amply described in recent papers ${ }^{29,32}$ and only a summary of the results, shown in Fig. 3, is given here. At room temperature the chemical capacitance is well described by
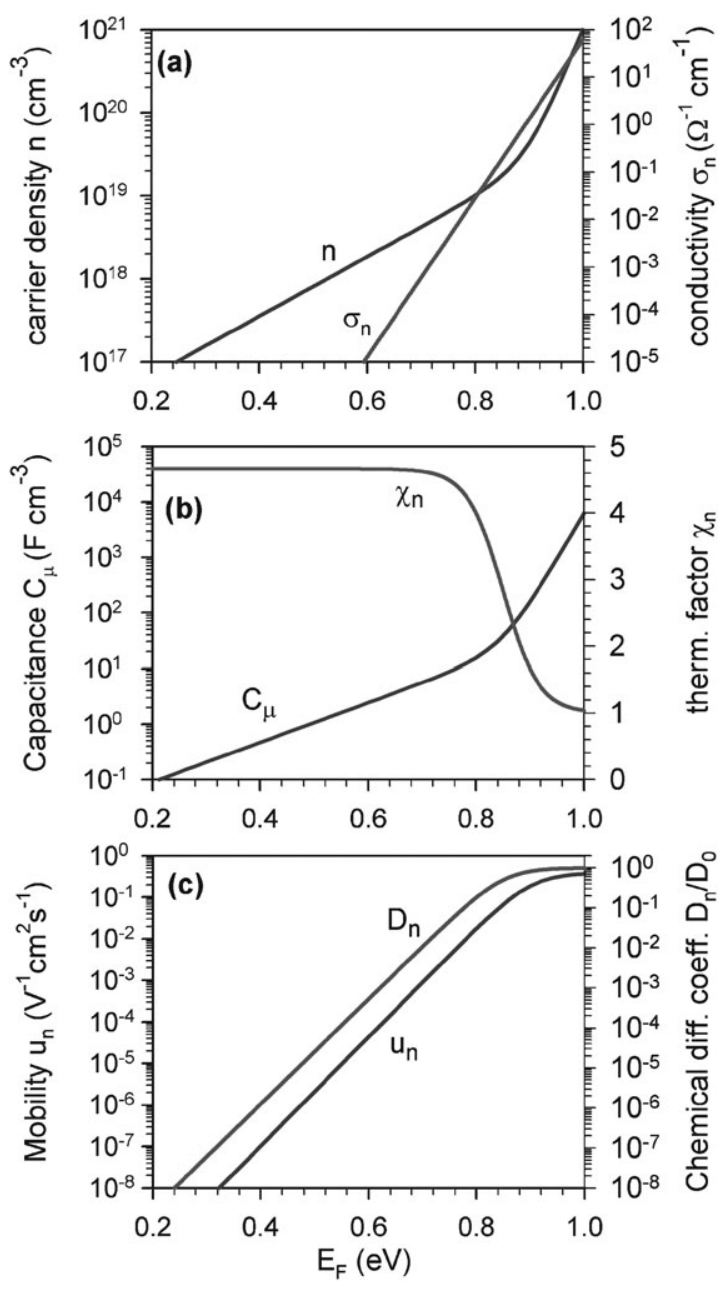

Fig. 3 Representation of several quantities for charge accumulation and transport by multiple trapping in an exponential DOS with the transport level at energy $E_{0}=1 \mathrm{eV} . E_{\mathrm{F}}$ is the Fermi level potential. (a) Carrier density and conductivity. (b) Chemical capacitance and thermodynamic factor. (c) Mobility and chemical diffusion coefficient. The following parameters were used in the calculation: $N_{0}=1.0 \times 10^{21}$ $\mathrm{cm}^{-3}, N_{1}=5.0 \times 10^{19} \mathrm{~cm}^{-3}, T=300 \mathrm{~K}, T_{0}=1400 \mathrm{~K}$, $\bar{D}_{0}=10^{-2} \mathrm{~cm}^{2} \mathrm{~s}^{-1}$. 
the approximation of eqn (6). Therefore we have

$$
C_{\mu}^{\mathrm{L}}=\frac{N_{\mathrm{L}} q^{2}}{k_{\mathrm{B}} T_{0}} \exp \left[\left(E-E_{0}\right) / k_{\mathrm{B}} T_{0}\right]
$$

with a slope $1 / k_{\mathrm{B}} T_{0}$ in log-linear representation shown in Fig. 3b. Note that the model is valid only for a deep distribution such that $T / T_{0}<1$. From eqn (49), the exponential distribution has the following property

$$
n=\int_{-\infty}^{E_{\mathrm{F}}} C_{\mu}^{\mathrm{L}} \mathrm{d} E_{\mathrm{F}}=\frac{k_{\mathrm{B}} T_{0}}{q^{2}} C_{\mu}^{\mathrm{L}}
$$

Therefore, the thermodynamic factor in eqn (12) is constant, ${ }^{29}$

$$
\chi_{n}=T_{0} / T
$$

For the typical values of $T_{0}, \chi_{n} \approx 2-5$ at room temperature, as shown in Fig. 3b. The diffusion-mobility ratio is independent of temperature:

$$
\frac{D_{n}}{u_{n}}=\frac{k_{\mathrm{B}} T_{0}}{q}
$$

Eqn (52) has been derived by Ritter et al. using eqn (15). ${ }^{65} \mathrm{~A}$ similar result was obtained by Baranovskii et al. ${ }^{99,100}$ for hopping electrons in non-equilibrium conditions at low temperature. The Einstein relation for multiple trapping in exponential DOS has been analyzed by Nguyen and O'Leary. ${ }^{67,68}$ They also derived the result in eqn (51) and therefore explained the difference between mobility and diffusion coefficient in $a$-Si : $\mathrm{H}$ previously reported by $\mathrm{Gu}$ et al. ${ }^{62}$

The calculation of the chemical diffusion coefficient with eqn (33), gives

$$
\begin{aligned}
D_{n} & =\frac{C_{\mu}^{0}}{C_{\mu}^{\mathrm{L}}} \bar{D}_{0} \\
& =\frac{N_{0} T_{0}}{N_{\mathrm{L}} T} \exp \left[-\left(E_{0}-E_{\mathrm{F}}\right)\left(\frac{1}{k_{\mathrm{B}} T}-\frac{1}{k_{\mathrm{B}} T_{0}}\right)\right] \bar{D}_{0}
\end{aligned}
$$

According to eqn (52) the mobility and chemical diffusion coefficient display identical dependence on the Fermi level, see Fig. 3c. When approaching the conduction band, the chemical capacitance is dominated by the free carriers, eqn (40), the thermodynamic factor decays to the ideal value 1 , and the transport coefficients take the constant value for the free electrons; as already explained, in this domain all the traps have been saturated and we observe the kinetics of free electrons. The conductivity shown in Fig. 3a is unaffected by the presence of traps; it is governed only by the free carrier density, as discussed above.

Recently, this model has been extensively applied in nanostructured metal oxides for DSC, ${ }^{1,2,101,102}$ where the different features exposed in Fig. 3 (for the multiple trapping regime in which $n_{\mathrm{L}} \gg n_{0}$ ) have been repeatedly observed: the exponential dependence of the chemical capacitance ${ }^{33,53}$ and chemical diffusion coefficient on the Fermi level, ${ }^{28,103,104}$ the constant thermodynamic factor, ${ }^{30}$ and the dependence of conductivity only on free carrier density. ${ }^{103,105}$

As an illustration of this model we show in Fig. 4 the experimental results of chemical capacitance and diffusion
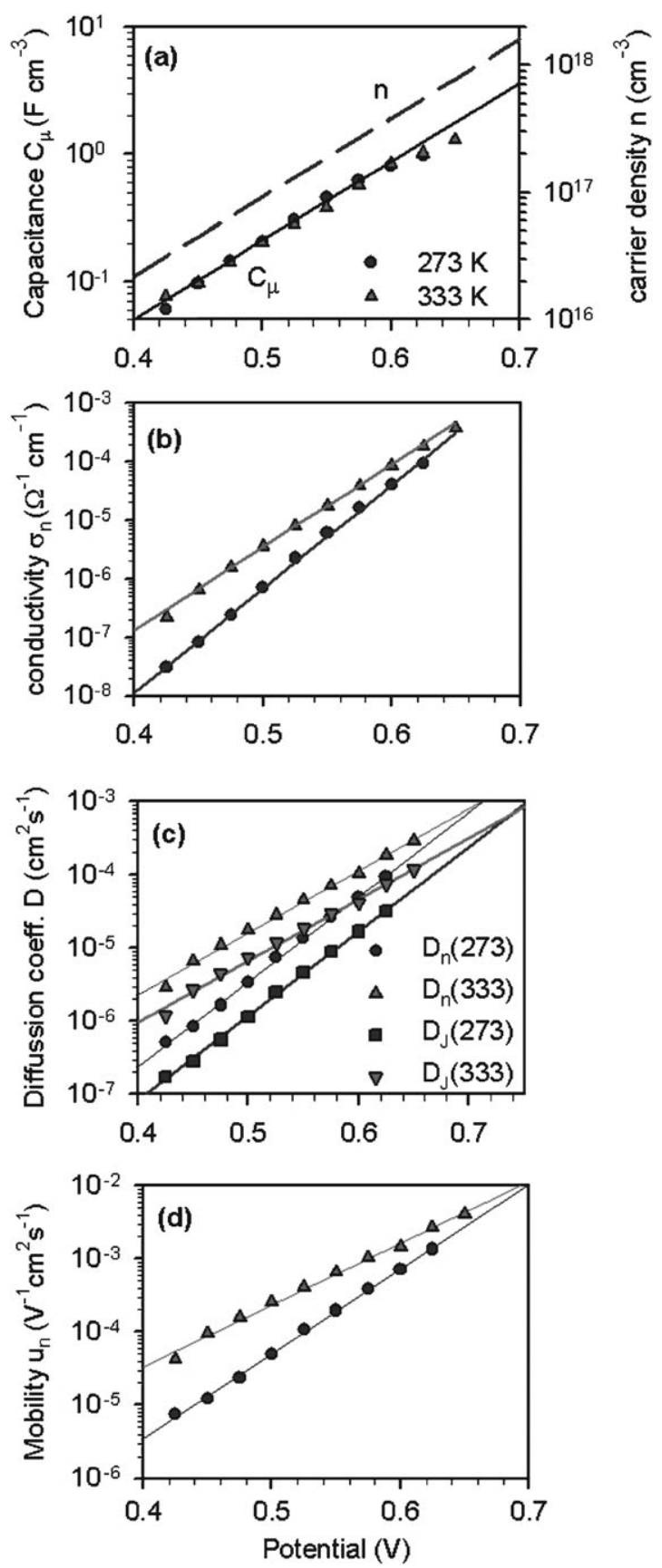

Fig. 4 Representation of several quantities for charge accumulation and transport at different temperatures, in a high efficiency $(10.2 \%)$ DSC. The experimental points are the chemical capacitance $C_{\mu}$ and conductivity $\sigma_{n}$, that are obtained from IS data on capacitance and transport resistance reported in ref. 103 , using the cell area $0.18 \mathrm{~cm}^{2}$ and active nanocrystalline $\mathrm{TiO}_{2}$ electrode thickness $12 \mu \mathrm{m}$. (a) Chemical capacitance. The fit line is $\ln C_{\mu}=-8.70+\mathrm{V} / 0.0704$, corresponding to $T_{0}=808 \mathrm{~K}$. The carrier density is calculated with eqn (50). (b) Electron conductivity. The fit lines are $\ln \sigma_{n}^{273} \mathrm{~K}=$ $-34.6+\mathrm{V} / 0.0245, \ln \sigma_{n}^{333 \mathrm{~K}}=-28.9+\mathrm{V} / 0.0306$. (c) Chemical diffusion coefficient $D_{n}$ calculated with eqn (20), and jump diffusion coefficient $D_{\mathrm{J}}$ calculated with eqn (13) and $\chi_{n}^{273 \mathrm{~K}}=2.95, \chi_{n}^{333 \mathrm{~K}}=$ 2.42. The lines are guides to the eyes. (d) Mobility calculated with eqn (18); the lines are guides to the eyes. 
conductivity derived from IS measurement in ref. 103 at different temperatures. First, the chemical capacitance in Fig. 4a shows the characteristic exponential dependence and is independent on temperature, confirming that the capacitance measures the DOS at the Fermi level as indicated in eqn (6). In contrast to this, the conductivity in Fig. 4b shows a strong dependence of temperature. The fit to straight lines gives values close to the thermal energies, $k_{\mathrm{B}} T=0.0236$ and $0.0288 \mathrm{eV}$ at 273 and $333 \mathrm{~K}$, respectively. Therefore, the conductivity is thermally activated as expected from eqn (34), assuming that the free electrons diffusion coefficient, $\bar{D}_{0}$, depends weakly on the temperature. Despite this observation, the value of $\bar{D}_{0}$ cannot be clearly identified, due to uncertainties about the carrier transport mechanism above the measured potential range (i.e., band transport or hopping to the transport energy), ${ }^{103}$ and this is discussed in the next section. The rest of quantities: carrier density, chemical diffusion coefficient, jump diffusion coefficient, and mobility, are derived from the previous ones and are also shown in Fig. 4.

\subsection{Hopping in exponential DOS}

The hopping model in an exponential distribution of states was developed in connection with amorphous semiconductors. $^{21,22}$ The difference with multiple trapping is that the carriers move by direct transitions between the localized states of the distribution in eqn (48). The transition probabilities are given by the upward and downward jump rates

$$
\begin{gathered}
\nu_{\uparrow}=\bar{\nu}_{0} \exp \left[-2 \frac{r}{\alpha}-\frac{E_{j}-E_{i}}{k_{\mathrm{B}} T}\right](E j>E i), \\
\nu_{\downarrow}=\bar{\nu}_{0} \exp \left[-2 \frac{r}{\alpha}\right]\left(E_{j} \leq E_{i}\right)
\end{gathered}
$$

where $\bar{v}_{0}$ is the attempt-to-jump frequency, $r$ is the distance between sites, $\alpha$ is the localization radius, and $E_{j}, E_{i}$, are the energies of the target and starting sites, respectively.

The concept of transport energy, $E_{\mathrm{tr}}$, has already been discussed in the Introduction. ${ }^{20,21}$ The following derivation is presented in ref. 106 and 107. In equilibrium the transport is governed by the fastest hop of a charge carrier. The most probable upward jump corresponds to an optimized combination of the distance and energy difference, eqn (54). Let $a=N_{L}^{-1 / 3}$ be the mean distance between localized sites. The average distance for states below the energy $E_{1}$ is

$$
\begin{aligned}
\left\langle r\left(E_{1}\right)\right\rangle & =\left[\frac{4 \pi}{3} \int_{-\infty}^{E_{1}} g(E) \mathrm{d} E\right]^{-1 / 3} \\
& =\left(\frac{4 \pi}{3}\right)^{-1 / 3} \exp \left(-\frac{E_{1}-E_{0}}{3 k_{\mathrm{B}} T_{0}}\right) a
\end{aligned}
$$

Now one can find the energy that optimizes the upward jump rate $\nu_{\uparrow}$, and the result is that the fastest hops occur in the vicinity of the transport energy, given by

$$
E_{\mathrm{tr}}=E_{0}-\Delta E_{\mathrm{tr}}
$$

where

$$
\Delta E_{\mathrm{tr}}=3 k_{\mathrm{B}} T_{0} \ln \left[\frac{3 \alpha T_{0}}{2 a T}\left(\frac{4 \pi}{3}\right)^{1 / 3}\right]
$$

independently of the energy of the starting site. ${ }^{106}$ The average jump distance is

$$
\left\langle r\left(E_{\mathrm{tr}}\right)\right\rangle=\frac{3 T_{0}}{2 T} \alpha
$$

Recently, we have reported ${ }^{108}$ the calculation of the chemical diffusion coefficient for the exponential distribution in the transport energy approximation. The calculation uses two main ingredients of previous results: (i) the average jump frequency given by Baranovskii et al., ${ }^{106}$ and (ii) the averaging procedure used by Arkhipov et al. ${ }^{79}$ to calculate the jump diffusion coefficient. While the latter procedure has been criticized $^{109}$ (in terms of the percolation considerations that are commented on in section 7), it seems so far as the only available procedure to obtain an analytically closed expression of $D_{n}$ (without the unknown prefactors that appear with the percolation criterion ${ }^{110}$ ) that can be directly compared with the experimental results. The result of our calculation gives ${ }^{108}$ the mean jump frequency

$$
\langle\nu\rangle=\nu_{0}\left(1-\frac{T}{T_{0}}\right) \exp \left[-3 \frac{T_{0}}{T}-\left(E_{\mathrm{tr}}-E_{\mathrm{F}}\right)\left(\frac{1}{k_{\mathrm{B}} T}-\frac{1}{k_{\mathrm{B}} T_{0}}\right)\right]
$$

So the chemical diffusion coefficient is

$$
\begin{aligned}
D_{n} & =\chi_{n}\left\langle r^{2}\left(E_{\mathrm{tr}}\right)\right\rangle\langle\nu\rangle \\
& =\frac{9 T_{0}^{3}}{4 T^{3}}\left(1-\frac{T}{T_{0}}\right) \exp \left[-3 \frac{T_{0}}{T}-\left(E_{\mathrm{tr}}-E_{F}\right)\left(\frac{1}{k_{\mathrm{B}} T}-\frac{1}{k_{\mathrm{B}} T_{0}}\right)\right] \alpha^{2} \nu_{0}
\end{aligned}
$$

By comparison with eqn (53), this last result shows, as expected, that in quasi-equilibrium conditions the hopping transport behaves in a similar way to multiple trapping, with the transport energy playing the role of the extended states level $E_{0}$. All these approximations require that the Fermi level is well below the transport energy.

The application of eqn (60) to the experimental results in Fig. 4 shows ${ }^{108}$ that the hopping model gives reasonable materials parameters and also explains the low situation of transport level that was already observed in previous experimental work. ${ }^{111}$ However, since multiple trapping and hopping model give similar results, it was concluded ${ }^{108}$ that more experimental work is needed, especially in the high carrier density regime, to clarify the transport mechanism in nanostructured $\mathrm{TiO}_{2}$ surrounded with liquid electrolyte.

\section{Carrier transport in organic conductors}

\subsection{General properties of the mobility}

Carrier transport in disordered organic materials has attracted a lot of interest in recent years in relation with new applications such as OLEDs and organic electronic devices. Charge carrier transport in molecular and organic materials is dominated by charge localization resulting from polarization of the medium and relaxation of molecular ions, and transport occurs via a sequence of charge-transfer steps from one molecule to another. Even in conjugated polymers with wellordered chains, macroscopic transport is impossible unless the carrier can hop to avoid the chain break and defects. ${ }^{112}$ Most 
work on transport in disordered organic conductors follows a Gaussian disorder model developed by Bässler ${ }^{14}$ based on hopping sites with a Gaussian distribution of site energies

$$
g\left(E, E_{1}\right)=\frac{N_{1}}{\sqrt{2 \pi} \sigma_{1}} \exp \left[-\frac{\left(E-E_{1}\right)^{2}}{2 \sigma_{1}^{2}}\right]
$$

where $E_{1}$ is the center of the distribution and $\sigma_{1}$ is the width.

Let us briefly discuss which are the basic observed features of the mobility in organic conductors. ${ }^{34}$ The mobility is obtained by different techniques (electrochemical, ${ }^{113-119}$ fieldeffect transistor, etc.), and the results may depend on the kind of measurement and preparation method, ${ }^{37}$ as shown in Fig. 5 for electrochemical measurement of two poly(3-hexylthiophene) films. ${ }^{120}$ In general, the conductivity and mobility in disordered organic materials show an extremely complex phenomenology, and the properties of electrochemically doped polymers often depend on the experimental conditions such as solvent and type of counterions. However, some regular properties have emerged. In most cases, the mobility shows a remarkable variation on Fermi level or carrier concentration, as seen in Fig. 5b. At low to moderate carrier density levels $u_{n}$ exhibits a constant (or decreasing) region, while at high carrier concentration, it first increases sharply, sometimes over several orders of magnitude, and later decreases. $^{113,114,120,121}$

These results can be described using models of increasing richness. First, the simple two-level model of section 4.2 already explains qualitatively the general features observed in the electrochemical measurement of the mobility of polarons in conducting polymers, ${ }^{113-119}$ see Fig. 2c. The single trap model also describes many features of doped organic crystals. ${ }^{11}$ However, it is widely agreed that disordered organic conductors present a Gaussian distribution as indicated in eqn (61), and this is in fact directly observed by capacitance measurements. ${ }^{122,123}$ Even the separate broadened contributions of polarons and bipolarons can be detected in the chemical capacitance. ${ }^{34}$ Therefore the next step is to use the multiple trapping model with the Gaussian distribution instead of a single trap level, ${ }^{124,125}$ and this will be described in section 6.2. It is also realized that organic conductors normally lack extended states as their inorganic counterparts. ${ }^{88}$ This requires to consider the hopping model, where the only possible carrier displacement mechanism consists of the transition between the localized states in the Gaussian distribution. This approach explains well the concentration dependence of the mobility, ${ }^{26,79}$ and will be discussed in section 6.3. In addition, it is important to discuss the effects of traps induced by impurities or doping, which requires to extend the DOS to a bimodal Gaussian. ${ }^{79,126}$ This type of model will be described in section 6.4 .

\subsection{Multiple trapping in the Gaussian DOS}

The multiple trapping model consisting of the Gaussian DOS of eqn (61) and a conduction band level at energy $E_{0}$ has mainly been used in connection with OLED devices. ${ }^{124,125}$ This model can be considered an extension of the two-level system of section 4.2 , by the introduction of disorder in the

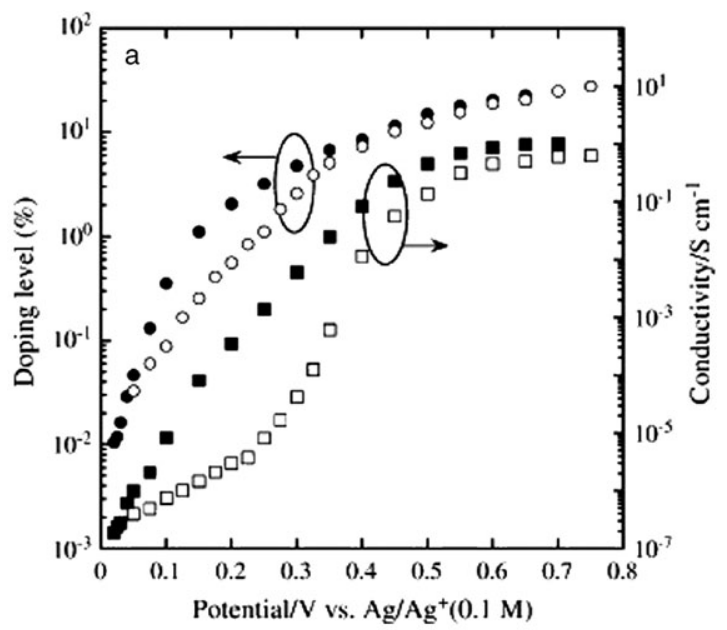

(a)

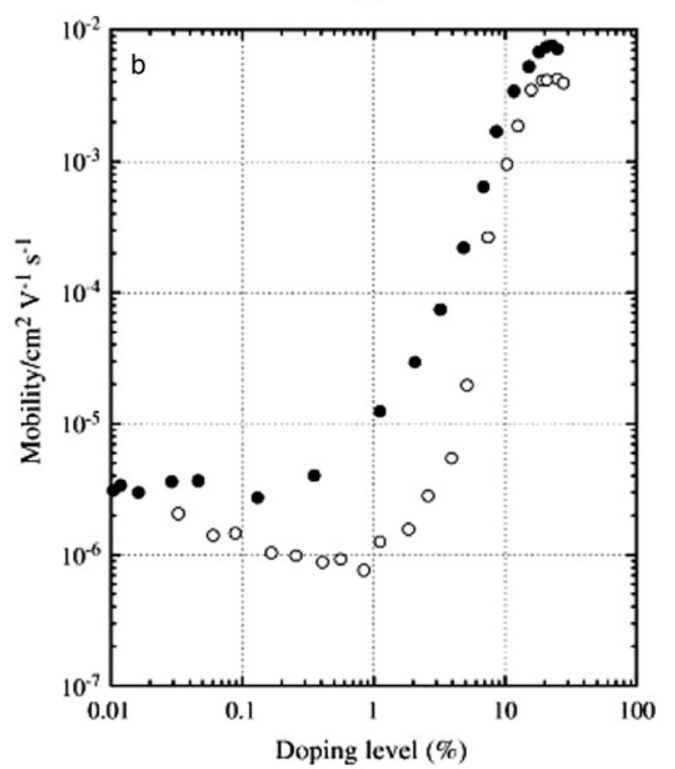

(b)

Fig. 5 (a) Potential dependences of doping levels and conductivities and (b) mobilities for two poly(3-hexylthiophene) films with identical chemical compositions. One of the films is obtained by electropolymerization of 3-hexylthiophene (as-grown film, $\boldsymbol{\square}$ ) and the other is prepared by casting a solution dissolving the as-grown film (cast film, ○, $\square$ ). Reprinted from Materials Letters, vol. 61, X. Jiang, Y. Harima and R. Patil, A transport study on as-grown and cast films of electrogenerated poly(3-hexylthiophene), p. 4687, Copyright (2007), with permission from Elsevier. ${ }^{120}$

trap. It is also interesting to discuss this model in detail because it provides a simple view of many features of the hopping model described later.

The results of our calculations, applying the general expressions derived in section 3.1, are shown in Fig. 6 and 7. For a detailed understanding of the behaviour of the transport coefficients it is important to review the properties of the carrier distribution in a Gaussian DOS. ${ }^{14}$ We compute the carrier distribution when the Fermi level is low enough that the occupancy is well described by Boltzman distribution. This 

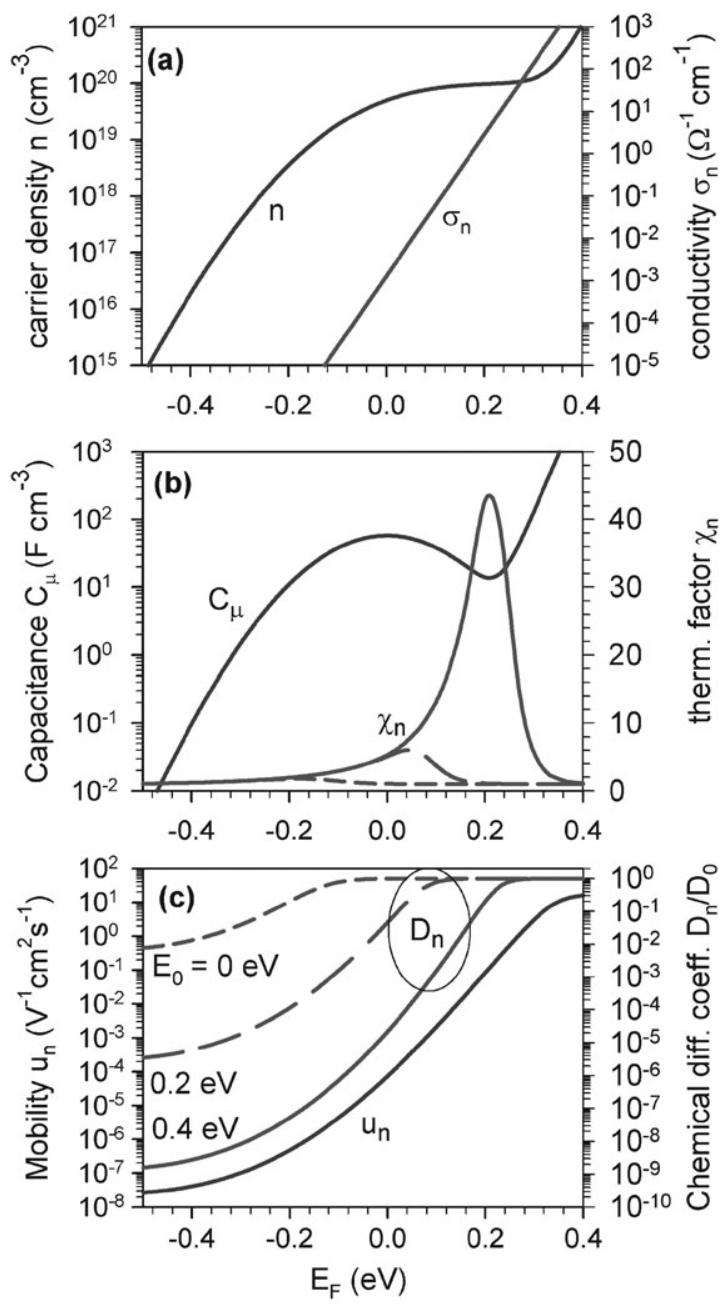

Fig. 6 Representation of several quantities for charge accumulation and transport by multiple trapping in a Gaussian DOS centered at $E_{1}=0 \mathrm{eV}$ with dispersion $\sigma_{1}=0.1 \mathrm{eV}$ and a transport level at energy $E_{0}=0.4 \mathrm{eV} . E_{\mathrm{F}}$ is the Fermi level potential. (a) Carrier density and conductivity. (b) Chemical capacitance and thermodynamic factor. (c) Mobility and chemical diffusion coefficient. In (b) and (c) the thermodynamic factor and chemical diffusion coefficient are shown also for different values of the transport level $E_{0}$, as indicated. The following parameters were used in the calculation: $N_{0}=1.0 \times 10^{21} \mathrm{~cm}^{-3}$, $N_{1}=1.0 \times 10^{20} \mathrm{~cm}^{-3}, T=300 \mathrm{~K}, \bar{D}_{0}=0.46 \mathrm{~cm}^{2} \mathrm{~s}^{-1}$.

domain corresponds to the region in which $\chi_{n} \approx 1$ in Fig. $6 \mathrm{~b}$. The carrier distribution is given by

$$
n_{\mathrm{L}}\left(E, E_{\mathrm{F}}\right)=g\left(E, E_{1}\right) \exp \left[-\left(E-E_{\mathrm{F}}\right) / k_{\mathrm{B}} T\right]
$$

By algebraic manipulation of eqn (62) we obtain

$$
n_{\mathrm{L}}\left(E, E_{\mathrm{F}}\right)=g\left(E, E_{m}\right) \exp \left[-\left(E_{\sigma}-E_{\mathrm{F}}\right) / k_{\mathrm{B}} T\right]
$$

where

$$
\begin{gathered}
E_{m}=E_{1}-\frac{\sigma_{1}^{2}}{k_{\mathrm{B}} T} \\
E_{\sigma}=E_{1}-\frac{\sigma_{1}^{2}}{2 k_{\mathrm{B}} T}
\end{gathered}
$$
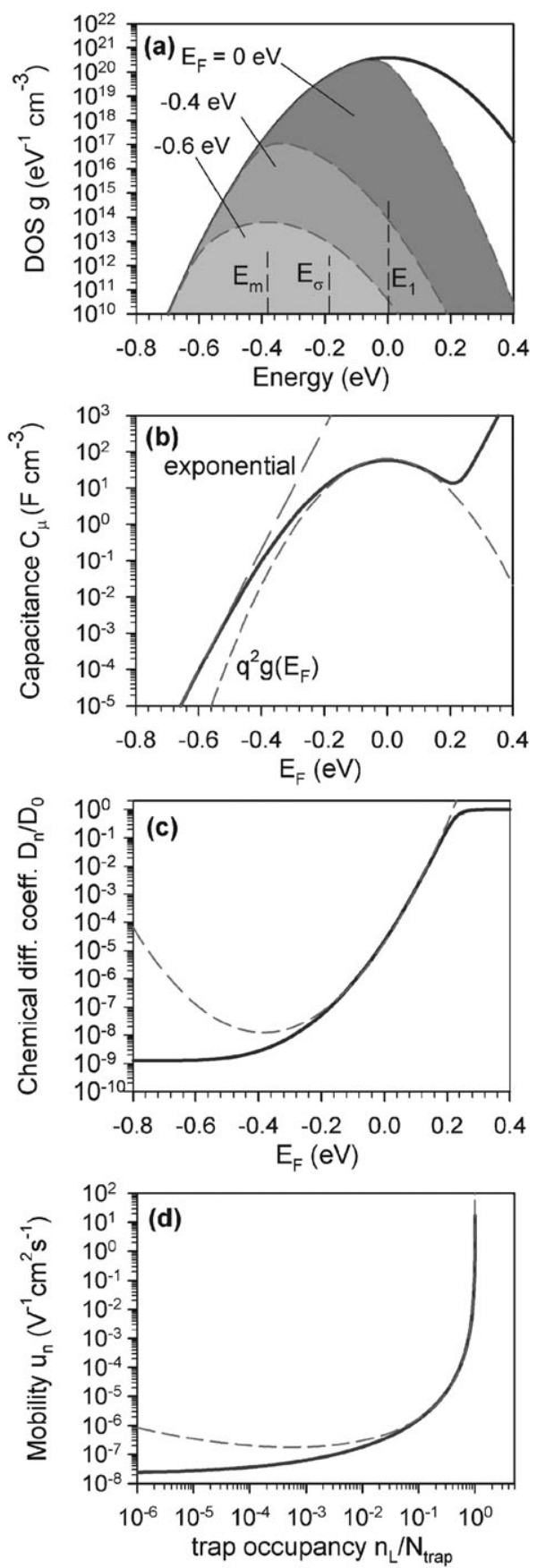

Fig. 7 Representation of several quantities for charge accumulation and transport by multiple trapping in a Gaussian DOS centered at $E_{1}=0 \mathrm{eV}$ with dispersion $\sigma_{1}=0.1 \mathrm{eV}$ and a transport level at energy $E_{0}=0.4 \mathrm{eV} . E_{\mathrm{F}}$ is the Fermi level potential. (a) Density of states. The dashed lines indicate the occupied states at different values of Fermi level, as indicated. (b) Chemical capacitance. The dashed lines are calculated from different approximation formulas as discussed in the main text. (c) Chemical diffusion coefficient. The dashed line is calculated with the approximation $D_{n}=\left[C_{\mu}^{0} / q^{2} g\left(E_{\mathrm{F}}\right)\right] \bar{D}_{0}$. (d) Mobility as a function of the occupation of the traps. The dashed line is calculated in the same approximation as in (c), using the thermodynamic factor. The following parameters were used in the calculation: $N_{0}=1.0 \times 10^{21} \mathrm{~cm}^{-3}, N_{1}=1.0 \times 10^{20} \mathrm{~cm}^{-3}$, $T=300 \mathrm{~K}, \bar{D}_{0}=0.46 \mathrm{~cm}^{2} \mathrm{~s}^{-1}$. 
According to eqn (63), when $E_{\mathrm{F}} \ll E_{m}$, the carriers form a Gaussian distribution of width $\sigma_{1}$ centered at energy level $E_{m}$, independently of the Fermi level. ${ }^{14,18,124}$ This is shown in Fig. 7a. In addition, using eqn (63) we obtain that the total carrier density in localized states is given by

$$
\begin{aligned}
n_{\mathrm{L}}\left(E_{\mathrm{F}}\right) & =\int_{-\infty}^{+\infty} n_{\mathrm{L}}\left(E, E_{\mathrm{F}}\right) \mathrm{d} E \\
& =N_{1} \exp \left[-\left(E_{\sigma}-E_{\mathrm{F}}\right) / k_{\mathrm{B}} T\right]
\end{aligned}
$$

According to eqn (66), the number of carriers in a Gaussian DOS when $E_{\mathrm{F}} \ll E_{m}$, is the same as in a monoenergetic level at $E_{\sigma}$ with total density $N_{1}$. Therefore at $E_{\mathrm{F}} \ll E_{m}$ the multiple trapping Gaussian model is identical to the two level (single trap) model of Fig. 2, taking $E_{\sigma}$ as the energy of the deep level. From eqn (66), the chemical capacitance has the value

$$
C_{\mu}^{\mathrm{L}}=\frac{q^{2} N_{1}}{k_{\mathrm{B}} T} \exp \left[-\left(E_{\sigma}-E_{\mathrm{F}}\right) / k_{\mathrm{B}} T\right]
$$

Therefore, when only the deep tail of the DOS is occupied, the capacitance is exponential, as indicated in Fig. 7b. Note that the zero-temperature approximation of eqn (6) (that requires that only states below the Ferrmi level are occupied) is invalid in this region in which the majority of carriers do not lie below the Fermi level, but instead, are above the Fermi level, ${ }^{124}$ symmetrically distributed around $E_{m}$, as shown in Fig. 7a.

Thermodynamic factors of the Gaussian distribution, as well as the implications for device modelling, have been amply discussed in the works of Roichman and Tessler ${ }^{66,72}$ and Peng et al. ${ }^{69,71,73}$

In Fig. $6 c$ the diffusion coefficient is calculated for different values of the transport level. It is observed that when the transport level is above the center of the Gaussian DOS, the diffusion coefficient and the mobility vary over many orders of magnitude. It is also observed that the variation of the diffusion coefficient is drastically modified by the position of the transport level.

Let us discuss the physical origin of the shape of the chemical diffusion coefficient. The saturation to a constant value at high Fermi level is due to the assumption of band transport, as before in Fig. 3c. However, in contrast with the exponential distribution, in the Gaussian case $D_{n}$ shows also a constant value at very low Fermi level in Fig. 6c. This is shown in more detail in Fig. 7c.

Using eqns (33), (40) and (67) we can obtain the chemical diffusion at very low concentration

$$
D_{n}=\frac{C_{\mu}^{0}}{C_{\mu}^{\mathrm{L}}} \bar{D}_{0}=\frac{N_{0}}{N_{1}} \exp \left[-\left(E_{0}-E_{\sigma}\right) / k_{\mathrm{B}} T\right] \bar{D}_{0}
$$

Eqn (68), which follows directly from the Hoesterey-Letson formula in eqn (47), gives the constant value of $D_{n}$ at low Fermi level observed in Fig. $6 \mathrm{c}$ and 7c. Since $\chi_{n}=1$ the constant mobility at low concentration is obtained from eqns (14) and (68). The transport coefficients are governed by thermal excitation between the effective trap level $E_{\sigma}$ and the transport level $E_{0}$. In Fig. $6 \mathrm{c}$ the changes of the lower limit of the diffusion coefficient were obtained by shifting the transport level; similar changes will be obtained by changing the disorder parameter $\sigma_{1}$, which modifies the level $E_{\sigma}$.

Our next step is to give an analytical expression for $D_{n}$ in the region where it rapidly increases between the two constant values at the extremes. In order to apply again eqn. (33), let us observe the features of the capacitance in Fig. 7b. When $E_{\mathrm{F}}>$ $E_{m}$, the zero temperature limit of the Fermi-Dirac function becomes a good approximation. Most of the carriers lie below the Fermi level, and eqn (6) describes well the capacitance, as is indicated in Fig. 7b. Therefore we can calculate an approximation to the chemical diffusion coefficient as

$$
\begin{aligned}
D_{n} & =\frac{C_{\mu}^{0}\left(E_{\mathrm{F}}\right)}{q^{2} g\left(E_{\mathrm{F}}, E_{1}\right)} \bar{D}_{0} \\
& =\frac{N_{0}}{N_{1}} \frac{\sqrt{2 \pi} \sigma_{1}}{k_{\mathrm{B}} T} \exp \left[\frac{\left(E_{\mathrm{F}}-E_{1}\right)^{2}}{2 \sigma_{1}^{2}}-\frac{E_{0}-E_{\mathrm{F}}}{k_{\mathrm{B}} T}\right] \bar{D}_{0}
\end{aligned}
$$

With algebraic manipulation of the exponent we obtain:

$$
D_{n}=\frac{N_{0}}{N_{1}} \frac{\sqrt{2 \pi} \sigma_{1}}{k_{\mathrm{B}} T} \exp \left[\frac{\left(E_{\mathrm{F}}-E_{m}\right)^{2}}{2 \sigma_{1}^{2}}-\frac{E_{0}-E_{\sigma}}{k_{\mathrm{B}} T}\right] \bar{D}_{0}
$$

The diffusion coefficient is a parabola (in a semilogarithmic plot) centered at $E_{m}$, shown in Fig. 7c in a dashed line. It is observed that eqn (70) provides a very good description of the steep increase of the chemical diffusion coefficient over many orders of magnitude. Using the thermodynamic factor and eqn (16), we also obtain from eqn (70) the rise of the mobility as a function of the concentration, shown in Fig. 7d.

In summary, all the properties of the chemical diffusion coefficient in a multiple trapping model with Gaussian disorder have been explained with simple considerations. Many of the properties are explained by the single trap model, taking into account the effective energies for carrier distribution and for carrier activation in eqns (64) and (65), respectively. The difference between the two models is that the rise of the chemical diffusion coefficient is linear (in a semilogarithmic representation) for the single trap, and parabolic for the Gaussian disorder.

\subsection{Hopping transport in the Gaussian DOS}

The mobility dependence on concentration in organic conductors has amply been studied in the last decade, on the basis of the hopping conduction in the Gaussian disorder model. ${ }^{18,25,26,79,126-130}$ Here we give a brief summary of the main trends of these models. We calculate the transport properties on the basis of the transport energy concept as formulated by Arkhipov et al. in a model that includes high carrier density effects. $^{79}$ This model and later developments ${ }^{26,130,131}$ constitute the most successful description available of the mobility dependence on concentration in electrochemically doped polymers. ${ }^{120}$ Similar results are obtained by direct solution of the master equation. ${ }^{128,132}$

As discussed above in the exponential distribution, in equilibrium conditions carriers most probably jump from the deep sites to a hopping site that belongs to the transport energy of the level $E_{\mathrm{tr}}$, which is determined by the 

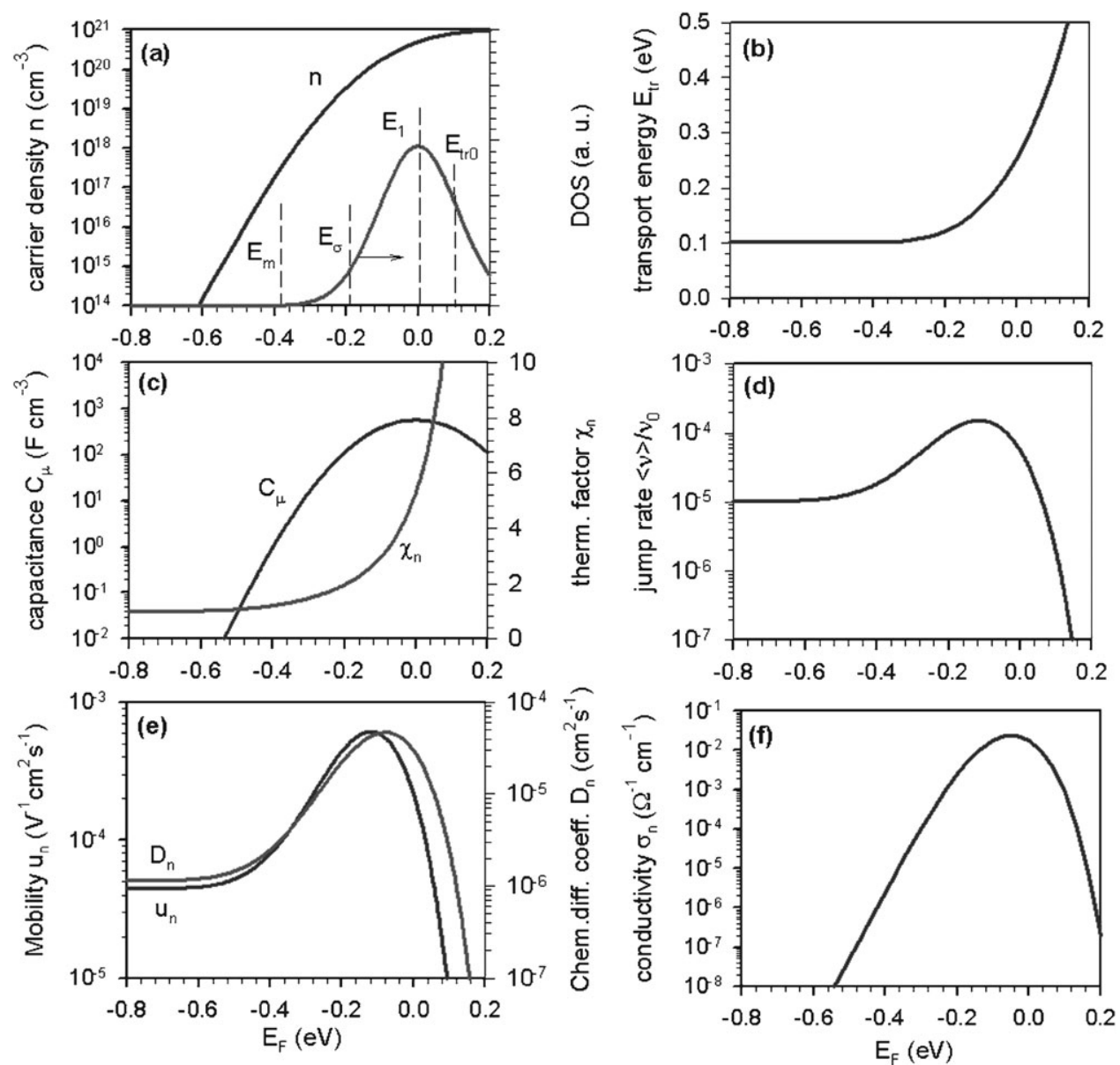

Fig. 8 Representation of several quantities for carrier accumulation and transport by hopping between localized states according to the transport energy concept, in a material with a Gaussian $\operatorname{DOS}\left(E_{1}=0 \mathrm{eV}, \sigma_{1}=0.1 \mathrm{eV}\right)$. $E_{\mathrm{F}}$ is the Fermi level potential. (a) Carrier density. Also shown is the DOS in linear scale. (b) Transport energy. (c) Chemical capacitance and thermodynamic factor. (d) Average jump frequency. (e) Mobility and chemical diffusion coefficient. (f) Conductivity. The following parameters were used in the calculation: $N_{1}=10^{21} \mathrm{~cm}^{-3}, T=300 \mathrm{~K}, \bar{v}_{0}=10^{13} \mathrm{~s}^{-1}$, $a=2 \times 10^{-8} \mathrm{~cm}$.

transcendental equation ${ }^{79}$

$$
\int_{-\infty}^{E_{\mathrm{tr}}} g(E)\left[1-f\left(E-E_{\mathrm{F}}\right)\right]\left(E_{\mathrm{tr}}-E\right)^{3} \mathrm{~d} E=\frac{6}{\pi}\left(\frac{k_{\mathrm{B}} T}{a}\right)^{3}
$$

The terms $g(E)(1-f)$ in the integrand of eqn (71) describe the density of vacant target sites for hopping. The average carrier jump rate is ${ }^{17}$

$$
\langle\nu\rangle=\frac{\bar{v}_{0}}{n} \int_{-\infty}^{E_{\mathrm{tr}}} g(E) f\left(E-E_{\mathrm{F}}\right) \exp \left(-\frac{E_{\mathrm{tr}}-E}{k_{\mathrm{B}} T}\right) \mathrm{d} E
$$

It has been pointed out ${ }^{109}$ that the jump frequency in eqn (72) should include the tunneling term $\exp (-2 r / \alpha)$, however here we maintain the original formulation of the model. ${ }^{79}$ The average square jump distance has the expression

$$
\left\langle r^{2}\right\rangle=\left[\int_{-\infty}^{E_{\mathrm{tr}}} g(E) \mathrm{d} E\right]^{-2 / 3}
$$

The jump diffusion coefficient can be calculated by eqn (8) as the product of eqns (72) and (73).

The results of this model are shown in Fig. 8 and 9, and two domains of behaviour can be distinguished. First at low carrier densities, the transport energy remains constant, Fig. 8b, and

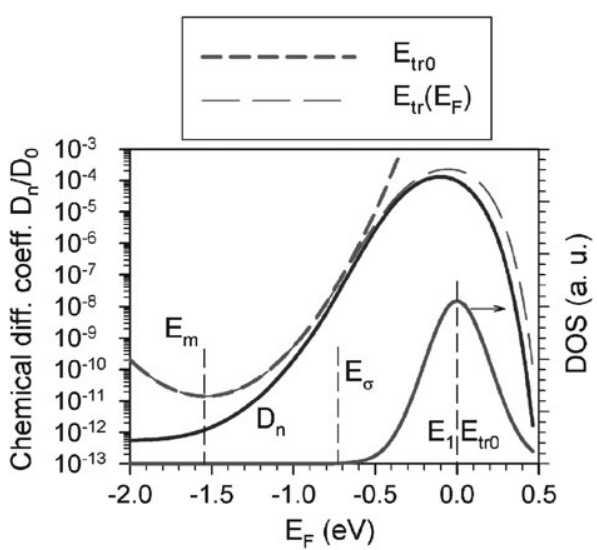

Fig. 9 Representation of the chemical diffusion coefficient as a function of the Fermi level potential $E_{\mathrm{F}}$, for transport by hopping between localized states according to the transport energy concept, in a material with a Gaussian DOS $\left(E_{1}=0 \mathrm{eV}, \sigma_{1}=0.2 \mathrm{eV}\right)$. Also shown is the DOS on a linear scale. The dashed lines are obtained with the approximation formula of multiple trapping, with a constant $\left(E_{\mathrm{tr} 0}=-0.04 \mathrm{eV}\right)$ and a variable value of the transport energy, $E_{\mathrm{tr}}$, as indicated. The following parameters were used in the calculation: $D_{0}=\bar{v}_{0} / N_{1}^{2 / 3}, N_{1}=10^{21} \mathrm{~cm}^{-3}$, $T=300 \mathrm{~K}, \bar{v}=10^{13} \mathrm{~s}^{-1}, a=2 \times 10^{-8} \mathrm{~cm}$. 
in this domain, as is well known, the occurrence of the effective transport level effectively reduces the hopping to multiple trapping, with $E_{\text {tr }}$ playing the role of the mobility edge. Therefore we obtain the characteristic behaviour of the chemical diffusion coefficient and mobility in multiple trapping, Fig. 8e, consisting of a constant value at $E<E_{m}$ and a rise when the tail of the DOS becomes occupied.

Second, at higher carrier densities, the transport level $E_{\mathrm{tr}}$ shifts upwards, ${ }^{79}$ see Fig. 8 b, due to the exclusion factor $(1-f)$ in eqn (71). Consequently, the average jump rate $\langle\nu\rangle$ declines, Fig. 8d, which causes a decrease of the transport coefficients, Fig. 8e, and also of the conductivity, Fig. $8 \mathrm{f}$. This behaviour is well documented in the electrochemical measurement of the mobility of conducting polymers. ${ }^{91,115,116,118,119,133}$ We remark that these features, due to the increasing localization of carriers by the full occupation of the DOS, were already described above in the single and two level systems, Fig. 1 and 2 , respectively.

It must also be noted that when the DOS is more than half occupied (i.e., at $E_{\mathrm{F}}>E_{1}$ ), the conditions of application of the transport energy concept are not justified, and the results plotted in Fig. 8 are only indicative. A more general treatment based on the effective medium approximation (EMA), including a very high carrier densities domain, has been presented recently, ${ }^{26}$ and the behaviour is qualitatively similar.

Let us consider the values at low concentration $\left(E<E_{m}\right)$ where the transport energy and chemical diffusion coefficient are constant, Fig. $8 \mathrm{~b}$ and e. Using the Boltzmann distribution for $f$ in eqn (72), and performing the integration as in eqn (66), we readily obtain

$$
\langle\nu\rangle=\bar{v}_{0} \exp \left[-\left(E_{t r}-E_{\sigma}\right) / k_{\mathrm{B}} T\right]
$$

If the transport level is well above the center of the Gaussian DOS, we may approximate $\left\langle r^{2}\right\rangle=N_{1}^{-2 / 3}$ with a very low error. Since the thermodynamic factor is $\chi_{n}=1$, the chemical diffusion coefficient has the value

$$
D_{n}=\frac{\bar{v}_{0}}{N_{1}^{2 / 3}} \exp \left[-\left(E_{\mathrm{tr}}-E_{\sigma}\right) / k_{\mathrm{B}} T\right]
$$

An equivalent expression of the mobility was given in ref. 17 .

We have remarked that hopping transport reduces to multiple trapping at low concentration. However, unlike in multiple trapping, there are no extended states in the hopping model, so the free carriers diffusion coefficient $\bar{D}_{0}$ is not defined a priori. Let us find the equivalent $\bar{D}_{0}$ in the hopping model, assuming that eqn (75) corresponds to the characteristic form of the diffusion coefficient in eqn (33). The chemical capacitance of the deep levels is given by eqn (67). On the other hand, the chemical capacitance of the "transport" level is

$$
C_{\mu}^{0}=\frac{q^{2} N_{1}}{k_{\mathrm{B}} T} \exp \left[-\left(E_{\mathrm{tr}}-E_{\mathrm{F}}\right) / k_{\mathrm{B}} T\right]
$$

Therefore, comparing eqns (75) and (33) we find

$$
\bar{D}_{0}=\frac{\bar{v}_{0}}{N_{1}^{2 / 3}}
$$

Following the same reasoning that led to eqn (70), we can calculate an approximation to the chemical diffusion coeffi- cient in the domain of increasing carrier density

$$
\begin{aligned}
D_{n} & =\frac{C_{\mu}^{0}\left(E_{\mathrm{F}}\right)}{q^{2} g\left(E_{\mathrm{F}}, E_{1}\right)} \bar{D}_{0} \\
& =\frac{\sqrt{2 \pi} \sigma_{1} \bar{\nu}_{0}}{k_{\mathrm{B}} T\left(N_{1}\right)^{2 / 3}} \exp \left[\frac{\left(E_{\mathrm{F}}-E_{m}\right)^{2}}{2 \sigma_{1}^{2}}-\frac{E_{\mathrm{tr}}\left(E_{\mathrm{F}}\right)-E_{\sigma}}{k_{\mathrm{B}} T}\right]
\end{aligned}
$$

In order to show more clearly this approximation, in Fig. 9 we plot the diffusion coefficient for a Gaussian of very large disorder, $\sigma_{1}=0.2 \mathrm{eV}$, which causes $D_{n}$ to vary over eight orders of magnitude. First we consider in eqn (78) a constant (low concentration) value of the transport energy, $E_{\mathrm{tr} 0}=-0.04 \mathrm{eV}$. Then $D_{n}$ is an inverted parabola centered at energy $E_{m}$, which gives a good agreement with the exact result at intermediate carrier densities, with a closed analytical expression. In Fig. 9 we also plot eqn (78) allowing for the upward shift of the transport energy, as in Fig. 8b. This gives a very good description $D_{n}$ also in the high carrier concentration domain.

\subsection{Hopping transport in the Gaussian DOS with traps}

The doping of disordered organic solids requires to consider two Gaussian distributions that overlap, since both the intrinsic transport states and the traps are affected by inhomogeneous broadening. The recent papers ${ }^{26,126}$ give an extensive account of the different behaviour of the mobility, according to the shape of the distribution, and the dominant transport mechanism (i.e., trap-controlled, trap-to-trap hopping, etc.). Also significant is the change of the DOS by coulomb interactions. The initial mobility decrease in electrochemical measurements ${ }^{37}$ shown in one of the samples in Fig. 5b is explained in terms of deep Coulomb traps created by doping. ${ }^{130,131}$

We assume that the DOS has the form

$$
\begin{aligned}
g(E)= & \frac{N_{1}}{\sqrt{2 \pi} \sigma_{1}} \exp \left[-\frac{\left(E-E_{1}\right)^{2}}{2 \sigma_{1}}\right] \\
& +\frac{N_{\text {trap }}}{\sqrt{2 \pi} \sigma_{\text {trap }}} \exp \left[-\frac{\left(E-E_{\text {trap }}\right)^{2}}{2 \sigma_{\text {trap }}}\right]
\end{aligned}
$$

The first component with number density $N_{1}$ is the intrinsic Gaussian distribution of the organic material. The second component with density $N_{\text {trap }}=\delta N_{1}$ is a deeper level, also with a Gaussian distribution, that acts in a similar way as traps. The results of our calculation using eqns (71)-(73) are shown in Fig. 10.

By introducing a small density of traps $(\delta=0.01)$ at level $E_{2}<E_{1}$, it is observed that the mobility at low carrier density decreases by one order of magnitude, while the conductivity does not change at all. This is the common behaviour of multiple trapping already discussed in previous sections; the lowering of the mobility is compensated in the conductivity by the increased number of carriers at traps. Introducing more abundant $(\delta=$ 0.1 ) and deeper traps at level $E_{3}$ causes some important modifications. First in Fig. 10b we appreciate that the traps shift downwards the transport energy ${ }^{8}$ so that the center of the intrinsic DOS becomes the transport level at low carrier densities. Since now the carriers hop to a deeper (and higher density) site than in the trap-free case, there is an increase of the conductivity at low densities. This is because the traps serve as 

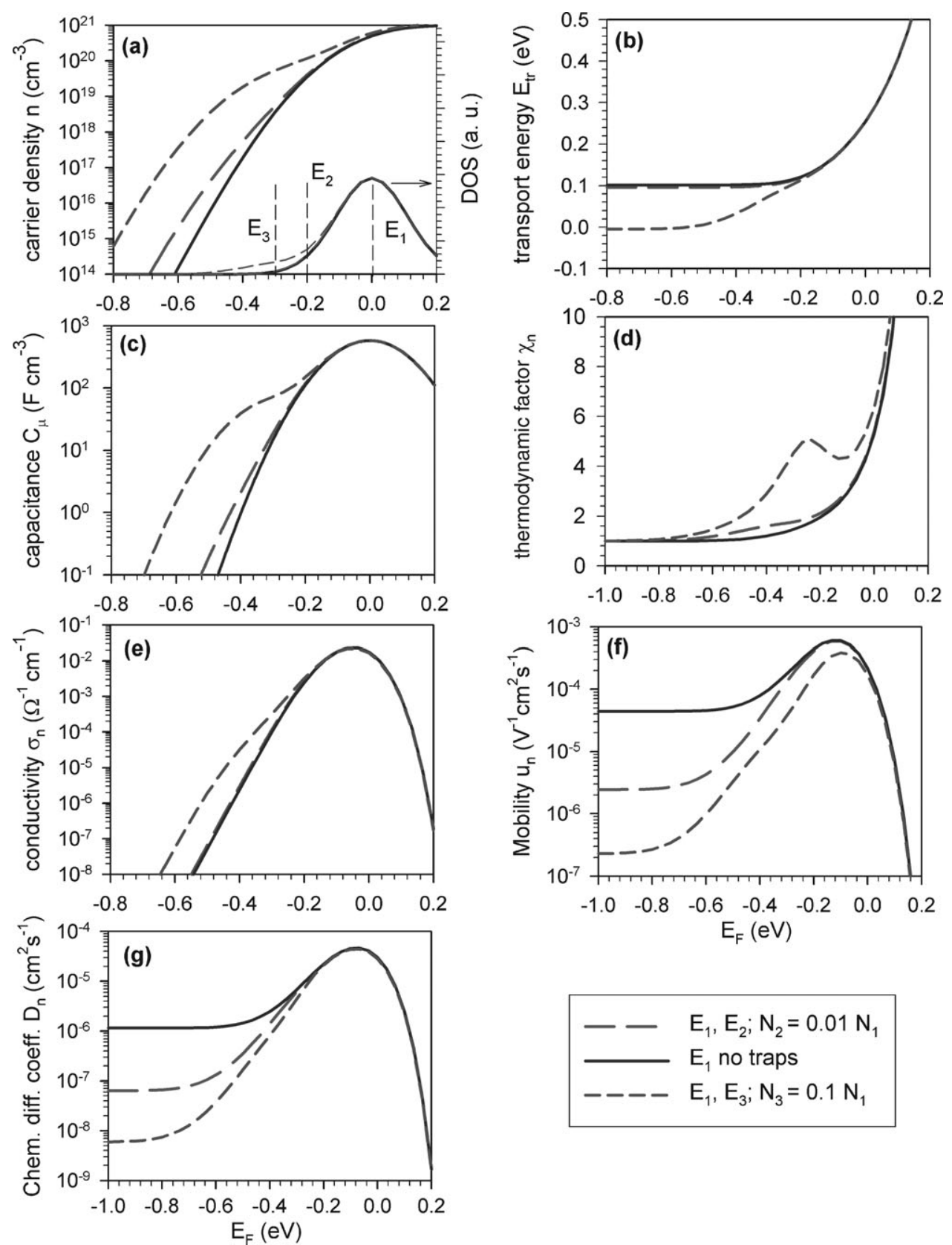

Fig. 10 Representation of several quantities for carrier accumulation and transport by hopping between localized states according to the transport energy concept, in a material with a Gaussian $\operatorname{DOS}\left(E_{1}=0 \mathrm{eV}, \sigma_{1}=0.1 \mathrm{eV}\right)$, and two different traps distribution, as indicated: $E_{2}=$ $-0.2 \mathrm{eV}, \sigma_{2}=0.1 \mathrm{eV}$ and $E_{3}=-0.3 \mathrm{eV}, \sigma_{3}=0.1 \mathrm{eV}$. $E_{\mathrm{F}}$ is the Fermi level potential. (a) Carrier density. Also shown are the different DOS in linear scale. (b) Transport energy. (c) Chemical capacitance. (d) Thermodynamic factor. (e) Conductivity. (f) Mobility and (g) chemical diffusion coefficient. The following parameters were used in the calculation: $N_{1}=10^{21} \mathrm{~cm}^{-3}, T=300 \mathrm{~K}, \bar{v}=10^{13} \mathrm{~s}^{-1}, a=2 \times 10^{-8} \mathrm{~cm}$.

an effective transport band. ${ }^{8}$ In addition, the mobility changes by three orders of magnitude, which is due to the larger separation between the trap and transport level, compared to the previous case. The enhancement of the mobility with carrier density in the presence of traps was previously demonstrated by numerical calculation based on the master equation. ${ }^{134,135}$

\section{Percolation}

In a system where carrier transport occurs by transitions between localized sites (or regions), with variable connectivity between the sites/regions, there are formed clusters of highly connected domains, which may or not be connected to surrounding clusters. There exists a threshold condition where a cluster of infinite size occurs, and current can percolate across the sample, and it is called the percolation threshold. ${ }^{136-138}$

Percolation is significant not only because of the onset of dc conduction at certain critical concentration. In addition, above the critical concentration the carriers will find out the least costly pathway in terms of activated transitions. Therefore, in the presence of widely varying local conductances, conduction may occur in far from spatially homogeneous conditions. 
Percolation effect is very significant in the theory of hopping conduction, where the difference in energy between hopping sites translates into widely variable timescales for the possible transitions. Therefore, the actual conduction path is determined by the faster connections that allow to obtain the percolation pathway, since the addition of slower connections does not contribute much to long range transport. ${ }^{12,13,19,139}$

The effect of percolation features prominently in the electron transport through redox centers in a solid matrix where the center concentration can be readily varied. ${ }^{7,36,140-142}$ However, in these systems the redox centers undergo displacement to a certain extent, from bounded motion around fixed positions, to long range diffusion. The electron transport is a combination of redox center mobility and electron hopping between centers. The work of Blauch and Saveant ${ }^{36}$ showed that the physical motion can eliminate completely the critical behaviour observed for static percolation.

A recent development of transport by redox exchange is the functionalization of nanostructured metal-oxides, and consists of a molecular layer adsorbed in the semiconductor surface that serves as an electronic transport relay by redox transitions between neighbour molecules. It was shown that the molecular layer conductivity can be changed between $\mathrm{n}$ and $\mathrm{p}$ characteristics, depending on the applied bias potential. ${ }^{143,144}$

Due to the widespread application of random nanoparticulate semiconductor networks in DSC and related systems, there has been recent interest in establishing a relationship between the geometry of the nanoparticulate array on the macroscopic electron transport. ${ }^{145-150}$ Lagemaat et al. first considered the relation between particle coordination number and porosity in $\mathrm{TiO}_{2}$ nanoparticulate films and described macroscopic transport in terms of the percolation model. ${ }^{145,146}$ To further investigate these properties, a unique model system of nanoporous $\mathrm{TiO}_{2}$ prepared by electrophoretic deposition (EPD) technique has been reported, where a systematic change of the porosity is possible, reducing the thickness of the film by pressing of deposited electrodes at different pressures. ${ }^{150,151}$ For this system, it has been shown ${ }^{152}$ that the electron diffusion coefficient follows well the Effective Medium Approximation model ${ }^{136,153}$ for electron percolation in random resistor netwroks, over a wide range of porosities.

\section{Conclusions}

The carrier transport properties in nanocrystalline semiconductors and organic materials are dominated by thermal activation to a band of extended states (multiple trapping), or if these do not exist, by hopping via localized states. In quasiequilibrium conditions, some thermodynamic quantities determined by the properties of the density of states (DOS) are crucial for the interpretation of transport properties. One is the chemical capacitance, $C_{\mu}$, that describes the step occupation by a small variation of the Fermi level. The other is the thermodynamic factor, $\chi_{n}$, that takes into account the deviation from Maxwell-Boltzmann statistics. It is also found necessary to distinguish carefully between two different forms of the diffusion coefficient. The chemical diffusion coefficient, $D_{n}$, is the normal form employed in Fick's law, and is therefore the universal coefficient used in the interpretation of measure- ments. The jump (or kinetic) diffusion coefficient, $D_{\mathrm{J}}$, relates to the single particle random walk. Both forms of the diffusion coefficient are connected as $D_{n}=\chi_{n} D_{\mathrm{J}}$. Consequently there are two (equivalent) forms of the generalized Einstein relation for the mobility to the diffusion ratio: one, using $D_{n}$, requires the inclusion of the thermodynamic factor, while the other one, using $D_{\mathrm{J}}$, does not, implying that the carrier mobility is proportional to the jump diffusion coefficient. This observation has consequences both for interpretation of measurements and for calculation with advanced transport models.

A common feature in the range of models reviewed, is that most properties of the chemical diffusion coefficient $D_{n}$ can be deduced from the chemical capacitance of the separate states involved in electron transport. This approach allows us to find a very simple interpretation of $D_{n}$ in complex models such as the hopping in a Gaussian DOS. In a multiple trapping scheme both transport coefficients $D_{n}$ and $u_{n}$ decrease (at a fixed Fermi level) by the introduction of more traps in the systems, while the conductivity $\sigma_{n}$ does not decrease by the effect of traps, provided that trapping-detrapping kinetics is fast.

\section{Appendix: Derivation of the Einstein relation}

We consider the motion of electrons in an organic or inorganic semiconductor material, with concentration $n(x)$ and electric field $F(x)=-\partial \phi / \partial x$ at position $x$. The electrical current is given by the sum of conduction and diffusion currents

$$
j_{n}=q n u_{n} F+q D_{n} \frac{\partial n}{\partial x}
$$

The electrochemical potential of electrons is $E_{\mathrm{F}}=-q \phi+\mu_{n}$. At equilibrium we have $\partial E_{F} / \partial x=0$ and consequently

$$
q \frac{\partial \phi}{\partial x}=\frac{\partial \mu_{n}}{\partial x}
$$

Therefore eqn (A1) gives

$$
j_{n}=n\left(-u_{n}+q D_{n} \frac{1}{n} \frac{\partial n}{\partial \mu_{n}}\right) \frac{\partial \mu_{n}}{\partial x}
$$

and using eqn (12)

$$
j_{n}=n\left(-u_{n}+\frac{q D_{n}}{k_{\mathrm{B}} T \chi_{n}}\right) \frac{\partial \mu_{n}}{\partial x}
$$

Since the current at equilibrium is $j_{n}=0$, the expression in parentheses in eqn (A4) is zero. This imposes a relationship, eqn (16), between the mobility and the chemical diffusion coefficient. Eqn (A1) can then be written in terms of the thermodynamic driving force

$$
j_{n}=-n u_{n} \frac{\partial E_{\mathrm{F}}}{\partial x}
$$

for the diffusion-drift displacement in quasi-equilibrium.

This derivation is clearly explained in the paper by Landsberg, ${ }^{56}$ and in terms of the chemical diffusion coefficient in ref. 32. In the work of Gomer ${ }^{48}$ it is stated that eqn (14) involves the chemical diffusion coefficient, contrary to our statement in eqn (18). This is because Gomer makes use of the Boltzmann statistics in his derivation, which reduces the validity to the 
cases in which $\chi_{n}=1$, and then eqns (16) and (18) are identical.

A decade ago, the occurrence of deviations from the standard Einstein relation in disordered systems with hopping carrier transport, ${ }^{58,62}$ caused some concern for the correctness of the results of hopping theories, which universally use eqn (14) for the calculation of mobilities. ${ }^{63,99}$ However this conflict is removed by the observation that hopping theories use eqn (18) and are compatible with experimental deviations from eqn (14). This is because the jump diffusion coefficient is not measured in diffusion experiments, that are usually described by Fick's law, and consequently by the chemical diffusion coefficient. Therefore, experimental quantities are related via the generalized Einstein relationship eqn (16).

Another approach to the Einstein relation in systems with trap-controlled transport is presented in a recent paper. ${ }^{70}$ The authors define the transport coefficient and the Einstein relation, in terms of the free carriers only. Therefore, the thermodynamic factor is 1 and the classical Einstein relation, eqn (14), is satisfied in different types of distributions of localized states. This is a trivial statement and we conclude that this approach $^{70}$ is not a proper analysis of multiple trapping transport. In experiments, carrier density and transport coefficients are strongly influenced by traps, that often have the dominant contribution on measured quantities. This is evident in many papers quoted in the present work. Furthermore, it is often not possible to separately determine the free electrons diffusion coefficient. This is the case in nanocrystalline $\mathrm{TiO}_{2}$ as shown here in Fig. 4; note that the band transport indicated in the model of Fig. 3 is not reached. ${ }^{103}$ Therefore in the main text we have used the definitions that are connected to experimentally measured quantities, as others have done. ${ }^{66-68}$

\section{Acknowledgements}

The work was supported by MEC projects MAT2007-62982 and Consolider HOPE CSD2007-00007, and GV project ACOMP07/03.

\section{References}

1 B. O'Regan and M. Grätzel, Nature, 1991, 353, 737.

2 J. Bisquert, D. Cahen, S. Rühle, G. Hodes and A. Zaban, J. Phys. Chem. B, 2004, 108, 8106

3 C. J. Brabec, N. S. Sariciftci and J. C. Hummelen, Adv. Mater., 2001, 11, 15

4 P. Peumans, U. Uchida and S. R. Forrest, Nature, 2003, 425, 158.

5 J. H. Burroughes, D. D. C. Bradley, A. R. Brown, R. N. Marks, K. MacKay, R. H. Friend, P. L. Burn and A. B. Holmes, Nature, 1990, 347, 539.

6 S. R. Forrest, Nature, 2004, 428, 911.

7 A. Heller, Curr. Opin. Chem. Biol., 2006, 10, 664

8 V. I. Arkhipov, E. V. Emelianova, P. Heremans and G. J. Adriaenssens, J. Optoelectr. Adv. Mater., 2002, 4, 425.

9 T. Tiedje and A. Rose, Solid State Commun., 1981, 37, 49.

10 J. Orenstein and M. Kastner, Phys. Rev. Lett., 1981, 46, 1421.

11 D. C. Hoesterey and G. M. Letson, J. Phys. Chem. Solids, 1963, 24, 1609 .

12 B. I. Shklovskii and A. L. Efros, Electronic Properties of Doped Semiconductors, Springer, Heidelberg, 1984.

13 H. Böttger and V. V. Bryksin, Hopping Conduction in Solids, Akademie Verlag, Berlin, 1985.

14 H. Bässler, Phys. Status Sol., B, 1993, 175, 15.
15 S. D. Baranovskii, H. Cordes, F. Hensel and G. Leising, Phys. Rev. B, 2000, 62, 7934.

16 S. D. Baranovskii, I. P. Zvyagin, H. Cordes, S. Yamashi and P. Thomas, Phys. Status Sol., B, 2002, 230, 281.

17 V. I. Arkhipov, E. V. Emelianova and G. J. Adriaenssens, Phys. Rev. B, 2001, 64, 125125 .

18 R. Coehoorn, W. F. Pasveer, P. A. Bobbert and C. J. Michels, Physical Review B, 2005, 72, 155206.

19 A. Miller and S. Abrahams, Phys. Rev., 1960, 120, 745.

20 M. Grünewald and P. Thomas, Phys. Status Sol., B, 1979, 94, 125 .

21 D. Monroe, Phys. Rev. Lett., 1985, 54, 146.

22 F. R. Shapiro and D. Adler, J. Non-Cryst. Solids, 1985, 74, 189.

23 S. D. Baranovskii, P. Thomas and G. J. Andrianssens, J. NonCryst. Solids, 1995, 190, 283.

24 S. D. Baranovskii, T. Faber, F. Hensel and P. Thomas, J. Phys.: Condens. Matter, 1997, 9, 2699.

25 O. Rubel, S. D. Baranovskii and P. Thomas, Phys. Rev. B, 2004, 69, 014206.

26 I. I. Fishchuk, V. I. Arkhipov, A. Kadashchuk, P. Heremans and H. Bässler, Phys. Rev. B, 2007, 76, 045210 .

27 C. Tanase, E. J. Meijer, P. W. M. Blom and D. M. de Leeuw, Phys. Rev. Lett., 2003, 91, 216601.

28 A. C. Fisher, L. M. Peter, E. A. Ponomarev, A. B. Walker and K. G. U. Wijayantha, J. Phys. Chem. B, 2000, 104, 949.

29 J. Bisquert, J. Phys. Chem. B, 2004, 108, 2323.

30 J. van de Lagemaat, N. Kopidakis, N. R. Neale and A. J. Frank, Phys. Rev. B, 2005, 71, 035304.

31 J. Bisquert, in Synthesis, Properties and Applications of Oxide Nanomaterials, ed. J. A. Rodríguez and M. Fernández García, Wiley, Weinheim, 2007.

32 J. Bisquert, Phys. Chem. Chem. Phys., 2008, 10, 49.

33 J. Bisquert, F. Fabregat-Santiago, I. Mora-Seró, G. GarciaBelmonte, E. M. Barea and E. Palomares, Inorg. Chim. Acta, 2008, 361, 684-698.

34 Z. Pomerantz, A. Zaban, S. Ghosh, J.-P. Lellouche, G. GarciaBelmonte and J. Bisquert, J. Electroanal. Chem., 2008, 614, 49.

35 C. E. D. Chidsey and R. W. Murray, J. Phys. Chem., 1986, 90, 1479.

36 D. N. Blauch and J. M. Savéant, J. Am. Chem. Soc., 1992, 114, 3323.

37 F. Laquai, G. Wegner and H. Bässler, Philos. Trans. R. Soc. A, $2007, \mathbf{3 6 5}, 1472$.

38 C. Uebing and R. Gomer, J. Chem. Phys., 1991, 95, 7626.

39 A. V. Myshlyavtsev, A. A. Stepanov, C. Uebing and V. P. Zhdanov, Phys. Rev. B, 1995, 52, 5977.

40 A. van der Ven, G. Ceder, M. Asta and P. D. Tepesch, Phys. Rev. $B, 2001,64,184307$.

41 M. D. Levi, G. Salitra, B. Markovski, H. Teller and D. Aurbach, J. Electrochem. Soc., 1999, 146, 1279.

42 A. L. Roest, J. J. Kelly and D. Vanmaekelbergh, Phys. Rev. Lett., 2002, 89, 036801 .

43 J. van de Lagemaat, Phys. Rev. B, 2005, 72, 235319.

44 A. Sinha, A. K. Sharma, R. Barui, A. R. Ghatak, S. Bhattacharya and K. P. Ghatak, Physica B, 2007, 391, 141.

45 D. A. Reed and G. Ehrlich, Surf. Sci., 1981, 102, 588.

46 H. Rickert, Electrochemistry of Solids, Springer Verlag, Berlin, 1982.

47 W. Weppner and R. A. Huggins, J. Electrochem. Soc., 1977, 124, 1569.

48 R. Gomer, Rep. Prog. Phys., 1990, 53, 917.

49 C. J. Wen, B. A. Boukamp, A. J. H. Huggins and W. Weppner, J. Electrochem. Soc., 1979, 126, 2258

50 M. D. Levi, C. Wang, D. Aurbach and Z. Chvoj, J. Electroanal. Chem., 2004, 562, 187.

51 J. Jamnik and J. Maier, Phys. Chem. Chem. Phys., 2001, 3, 1668.

52 J. Bisquert, Phys. Chem. Chem. Phys., 2003, 5, 5360.

53 F. Fabregat-Santiago, I. Mora-Seró, G. Garcia-Belmonte and J. Bisquert, J. Phys. Chem. B, 2003, 107, 758.

54 C. Uebing and R. Gomer, J. Chem. Phys., 1994, 100, 7759.

55 L. S. Darken, Trans. Am. Inst. Min. Eng., 1948, 175, 184.

56 P. T. Landsberg, Eur. J. Phys., 1981, 2, 213.

57 P. N. Butcher, J. Phys. C: Solid State Phys., 1972, 5, 3164.

58 R. Richert, L. Pautmeier and H. Bässler, Phys. Rev. Lett., 1989, 63, 547. 
59 J. M. Casado and J. J. Mejias, Philos. Mag. B, 1994, 70, 1111. 60 S. D. Baranovskii, T. Faber, T. Hensel and P. Thomas, J. NonCryst. Solids, 1998, 227-230, 158.

61 P. M. Borsenberger, L. Pautmeier, R. Richert and H. Bässler, J. Chem. Phys., 1991, 94, 8276.

62 Q. Gu, E. A. Schiff, S. Grebner, F. Wang and R. Schwartz, Phys. Rev. Lett., 1996, 76, 3196.

63 S. D. Baranovskii, T. Faber and P. Thomas, Phys. Status Sol., B, 1998, 205, 87.

64 V. R. Nikitenko, H. von Seggern and H. Bässler, J. Phys.: Condens. Matter, 2007, 19, 136210.

65 D. Ritter, E. Zeldov and K. Weiser, Phys. Rev. B, 1988, 38, 8296.

66 Y. Roichman and N. Tessler, Appl. Phys. Lett., 2002, 80, 1948.

67 T. H. Nguyen and S. K. O'Leary, Appl. Phys. Lett., 2003, 83, 1998.

68 T. H. Nguyen and S. K. O'Leary, J. Appl. Phys., 2005, 98, 076102.

69 Y. Q. Peng, S. Sun and C. A. Song, Mater. Sci. Semiconduct. Process., 2005, 8, 525.

70 F. Neumann, Y. A. Genenko and H. von Seggem, J. Appl. Phys., 2006, 99, 013704.

71 Y. Q. Peng, J.-H. Yang and F.-P. Lu, Appl. Phys. A, 2006, 83, 305-311.

72 N. Tessler and Y. Roichman, Org. Electron., 2005, 6, 200-210.

73 Y. Q. Peng, J.-H. Yang, F.-P. Lu, Q.-S. Yang, H.-W. Xing, X.-S. Li and C.-A. Song, Appl. Phys. A, 2006, 86, 225.

74 S. D. Baranovskii and O. Rubel, in Charge Transport in Disordered Solids with Applications to Electronics, ed. S. D. Baranovskii, Wiley, Weinheim, 2006, p. 221.

75 N. W. Ashcroft and N. D. Mermin, Solid State Phys., Thomson Learning, Florence, 1976.

76 R. A. Smith, Semiconductors, Cambridge University Press, Cambridge, 1978.

77 I. I. Fishchuk, A. Kadashchuk, H. Bässler and S. Nespurek, Phys. Rev. B, 2003, 67, 224303.

78 V. I. Arkhipov, P. Heremans, E. V. Emelianova, G. J. Adriaenssens and H. Bässler, J. Phys.: Condens. Matter, 2002, 14, 9899-9911.

79 V. I. Arkhipov, P. Heremans, E. V. Emelianova, G. J. Adriaenssens and H. Bässler, Appl. Phys. Lett., 2003, 82, 3245.

80 J. Bisquert and V. S. Vikhrenko, J. Phys. Chem. B, 2004, 108, 2313.

81 N. F. Mott and E. A. Davies, Electronic Processes in NonCrystalline Materials, Clarendon Press, Oxford, 1971.

82 W. W. Focke and G. E. Wneck, J. Electroanal. Chem., 1988, 256, 343.

83 I. N. Hulea, H. B. Brom, A. J. Houtepen, D. Vanmaekelbergh, J. J. Kelly and E. A. Meulenkamp, Phys. Rev. Lett., 2004, 93, 166601.

84 E. W. Paul, A. J. Ricco and M. S. Wrighton, J. Phys. Chem., $1985,89,1441$.

85 D. Vanmaekelbergh, A. J. Houtepen and J. J. Kelly, Electrochim. Acta, 2007, 53, 1140.

86 J. Bisquert, J. Phys. Chem. B, 2002, 106, 325.

87 V. G. Kytin, J. Bisquert, I. Abayev and A. Zaban, Phys. Rev. B, 2004, 70, 193304.

88 R. Schmechel and H. von Seggern, Phys. Status Sol., A, 2004, 201, 1215 .

89 M. D. Levi, E. Markevich and D. Aurbach, Electrochim. Acta, 2005, 51, 98.

90 C. A. Niklasson and C.-G. Granqvist, J. Mater. Chem., 2007, 17, 127.

91 R. Patil, Y. Harima and X. Jiang, Electrochim. Acta, 2004, 49, 4687.

92 W. Shockley and W. T. Read, Phys. Rev., 1952, 87, 835.

$93 \mathrm{~J}$. Bisquert, manuscript in preparation.

94 A. Rose, RAC rev., 1951, 12, 362.

95 A. Rose, Concepts in Photoconductivity and Allied Problems, Interscience, New York, 1963.

96 S. E. Guidoni and C. M. Aldao, Eur. J. Phys., 2002, 23, 395.

97 D. Yu, C. Wang and P. Guyot-Sionnest, Science, 2003, 300, 1277.

98 J. Bisquert and V. S. Vikhrenko, Electrochim. Acta, 2002, 47, 3977.

99 S. D. Baranovskii, T. Faber, T. Hensel, P. Thomas and G. J. Adriaenssens, J. Non-Cryst. Solids, 1996, 198-200, 214.
100 V. I. Arkhipov and G. J. Adriaenssens, J. Phys.: Condens. Matter, 1996, 8, 7909.

101 L. M. Peter, Phys. Chem. Chem. Phys., 2007, 9, 2630.

102 L. M. Peter, J. Phys. Chem. C, 2007, 111, 6601.

103 Q. Wang, S. Ito, M. Grätzel, F. Fabregat-Santiago, I. Mora-Seró, J. Bisquert, T. Bosshoa and H. Imaic, J. Phys. Chem. B, 2006, 110, 19406.

104 Y. Fukai, Y. Kondo, S. Mori and E. Suzuki, Electrochem. Commun., 2007, 9, 1439.

105 I. Abayev, A. Zaban, F. Fabregat-Santiago and J. Bisquert, Phys. Status Sol., A, 2003, 196, R4.

106 S. D. Baranovskii, P. Thomas and G. J. Adriaenssens, J. NonCryst. Solids, 1995, 190, 283.

107 B. Hartenstein and H. Bässler, J. Non-Cryst. Solids, 1995, 190, 112.

108 J. Bisquert, J. Phys. Chem. C, 2007, 111, 17163.

109 S. D. Baranovskii, O. Rubel and P. Thomas, Thin Solid Films, 2005, 487, 2 .

110 M. C. J. M. Vissenberg and M. Matters, Phys. Rev. B, 1998, 57, 12964.

111 B. O'Regan and J. R. Durrant, J. Phys. Chem. B, 2006, 110, 8544.

112 R. S. Kohlman and A. J. Epstein, in Handbook of conducting polymers, ed. T. A. Skotheim, R. L. Elsenbaumer and J. R. Reynolds, Marcel Dekker Inc., New York, 1986, vol. 1, p. 85.

113 Y. Harima, D.-H. Kim, Y. Tsutitori, X. Jiang, R. Patil, Y. Ooyama, J. Ohshita and A. Kunai, Chem. Phys. Lett., 2006, 420, 387-390.

114 H. Shimotani, G. Diguet and Y. Iwasa, Appl. Phys. Lett., 2005, 86, 022104

115 Y. Harima, F. Ogawa, R. Patil and X. Jiang, Electrochim. Acta, 2007, 52, 3615.

116 T. Ohsawa, T. Kabata, O. Kimura, M. Onoda and K. Yoshino, Jpn. J. Appl. Phys., 1989, 28, 996.

117 D. Ofer, R. M. Crooks and M. S. Wrighton, J. Am. Chem. Soc., 1990, 112, 7869.

118 R. Patil, Y. Harima, K. Yamashita, K. Komaguchi, Y. Itagaki and M. Shiotani, J. Electroanal. Chem., 2002, 518, 13.

119 G. Zotti, S. Zecchin, B. Vercelli, A. Berlin, S. Grimoldi, M. C. Pasini and M. M. M. Raposo, Chem. Mater., 2005, 17, 6492.

120 X. Jiang, Y. Harima and R. Patil, Mater. Lett., 2007, 61, 4687.

121 H. J. Snaith and M. Grätzel, Phys. Rev. Lett., 2007, 98, 177402.

122 J. Bisquert, G. Garcia-Belmonte and J. García-Cañadas, J. Chem. Phys., 2004, 120, 6726.

123 J. García-Cañadas, F. Fabregat-Santiago, H. Bolink, E. Palomares, G. Garcia-Belmonte and J. Bisquert, Synth. Meth., 2006, 156, 944.

124 V. I. Arkhipov, P. Heremans, E. V. Emelianova and G. J. Adriaenssens, Appl. Phys. Lett., 2001, 79, 4154.

125 T. H. Nguyen, M. Schmeits and H. P. Loebl, Phys. Rev. B, 2007, 75, 075307.

126 R. Coehoorn, Phys. Rev. B, 2007, 75, 155203.

127 C. Tanase, P. W. M. Blom and D. M. de Leeuw, Phys. Rev. B, 2004, 70, 193202.

128 W. F. Pasveer, J. Cottar, C. Tanase, R. Coehoorn, P. A. Bobbert, P. W. M. Blom, D. M. de Leeuw and C. J. Michels, Phys. Rev. Lett., 2005, 94, 206601.

129 J. Zhou, Y. C. Zhou, J. M. Zhao, C. Q. Wu, X. M. Ding and X. Y. Hou, Phys. Rev. B, 2007, 75, 153201.

130 V. I. Arkhipov, E. V. Emelianova, P. Heremans and H. Bässler, Phys. Rev. B, 2005, 72, 235202.

131 V. I. Arkhipov, P. Heremans, E. V. Emelianova and H. Bässler, Phys. Rev. B, 2005, 72, 045214.

132 J. Cottar and P. A. Bobbert, Phys. Rev. B, 2006, 74, 115204

133 X.-L. Wei and A. J. Epstein, Synth. Meth., 1997, 84, 791.

134 Z. G. Yu, D. L. Smith, A. Saxena, R. L. Martin and A. R. Bishop, Phys. Rev. Lett., 2000, 84, 721.

135 Z. G. Yu, D. L. Smith, A. Saxena, R. L. Martin and A. R. Bishop, Phys. Rev. B, 2001, 63, 085202.

136 S. Kirkpatrick, Rev. Mod. Phys., 1973, 45, 574.

137 J. M. Ziman, Models of Disorder, Cambridge University Press, Cambridge, 1979.

138 M. Sahimi, Applications of Percolation Theory, CRC Press, Boca Raton, 1994.

139 S. D. Baranovskii, Charge Transport in Disordered Solids with Applications to Electronics, Wiley, Weinheim, 2006. 
140 J. S. Facci, R. H. Schmedl and R. W. Murray, J. Am. Chem. Soc., 1982, 104, 4959.

141 J. W. Long, C. S. Velazquez and R. W. Murray, J. Phys. Chem., 1996, 100, 5492.

142 S. Kaneko, Progr. Polym. Sci., 2001, 26, 1101.

143 Q. Wang, S. M. Zakeeruddin, J. Cremer, P. Bäuerle, R. Humphry-Baker and M. Grätzel, J. Am. Chem. Soc., 2005, 127, 5706.

144 J. Bisquert, M. Grätzel, Q. Wang and F. Fabregat-Santiago, J. Phys. Chem. B, 2006, 110, 11284.

145 J. van de Lagemaat, K. D. Benkstein and A. J. Frank, J. Phys. Chem. B, 2001, 105, 12433.

146 K. D. Benkstein, N. Kopidakis, J. Van de Lagemaat and A. J. Frank, J. Phys. Chem. B, 2003, 107, 7759.
147 S. Tirosh, T. Dittrich, A. Ofir, L. Grinis and A. Zaban, J. Phys. Chem. B, 2006, 110, 16165.

148 M. J. Cass, F. L. Qiu, A. B. Walker, A. C. Fisher and L. M. Peter, J. Phys. Chem. B, 2003, 107, 113.

149 M. Ni, M. K. H. Leung, D. Y. C. Leung and K. Sumathy, Sol. Energy Mater. Sol. Cells, 2006, 90, 1331.

150 T. Dittrich, A. Ofir, S. Tirosh, L. Grinis and A. Zaban, Appl. Phys. Lett., 2006, 88, 182110.

151 A. Ofir, T. Dittrich, S. Tirosh, L. Grinis and A. Zaban, J. Appl. Phys., 2006, 100, 074317.

152 A. Ofir, S. Dor, L. Grinis, A. Zaban, T. Dittrich and J. Bisquert, J. Chem. Phys., 2008, 128, 064703.

153 J. Bernasconi and H. J. Wiesmann, Phys. Rev. B, 1976, 13, 1131. 